\title{
Cardiomyocyte-Specific Human Bcl2-Associated Anthanogene 3 P209L Expression Induces Mitochondrial Fragmentation, Bcl2-Associated Anthanogene 3 Haploinsufficiency, and Activates p38 Signaling
}

\author{
Megan T. Quintana, ${ }^{*}$ Traci L. Parry, ${ }^{\dagger}$ Jun He, ${ }^{\ddagger}$ Cecelia C. Yates, ${ }^{\S}$ Tatiana N. Sidorova, ${ }^{\llbracket}$ Katherine T. Murray, ${ }^{\varpi}$ James R. Bain, ${ }^{\| * *}$ \\ Christopher B. Newgard, ${ }_{* * *}$ Michael J. Muehlbauer, Samuel C. Eaton, ${ }^{\dagger \dagger}$ Akinori Hishiya, ${ }^{\dagger \dagger}$ Shin Takayama, ${ }^{\$ \delta}$ and Monte S. Willis ${ }^{\dagger \dagger \dagger \uparrow ब ~}$ \\ From the Departments of Surgery, ${ }^{*}$ Pharmacology, ${ }^{\dagger \dagger}$ and Pathology and Laboratory Medicine, ${ }^{\mathbf{\top}}$ and the McAllister Heart Institute, ${ }^{\dagger}$ University of North \\ Carolina, Chapel Hill, North Carolina; the General Hospital of Ningxia Medical University, ${ }^{\ddagger}$ Yinchuan, Ningxia, People’s Republic of China; the Department \\ of Health Promotions and Development, ${ }^{\S}$ School of Nursing, University of Pittsburgh, Pittsburgh, Pennsylvania; the Departments of Medicine and \\ Pharmacology, "Vanderbilt University School of Medicine, Nashville, Tennessee; the Sarah W. Stedman Nutrition and Metabolism Center," Duke Molecular \\ Physiology Institute, and the Division of Endocrinology, Metabolism, and Nutrition, ** Department of Medicine, Duke University Medical Center, Durham, \\ North Carolina; the Boston Strategics Corp. ${ }^{\ddagger \ddagger}$ Boston, Massachusetts; the Department of Pathology, ${ }^{\S \S}$ Boston University, Boston, Massachusetts
}

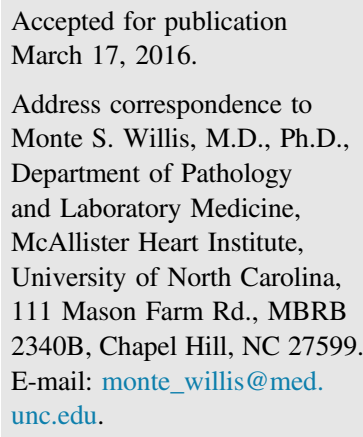

\begin{abstract}
The Bcl2-associated anthanogene (BAG) 3 protein is a member of the BAG family of cochaperones, which supports multiple critical cellular processes, including critical structural roles supporting desmin and interactions with heat shock proteins and ubiquitin ligases intimately involved in protein quality control. The missense mutation P209L in exon 3 results in a primarily cardiac phenotype leading to skeletal muscle and cardiac complications. At least 10 other Bag3 mutations have been reported, nine resulting in a dilated cardiomyopathy for which no specific therapy is available. We generated $\alpha \mathrm{MHC}-$ human Bag3 P209L transgenic mice and characterized the progressive cardiac phenotype in vivo to investigate its utility in modeling human disease, understand the underlying molecular mechanisms, and identify potential therapeutic targets. We identified a progressive heart failure by echocardiography and Doppler analysis and the presence of pre-amyloid oligomers at 1 year. Paralleling the pathogenesis of neurodegenerative diseases (eg, Parkinson disease), pre-amyloid oligomers-associated alterations in cardiac mitochondrial dynamics, haploinsufficiency of wild-type BAG3, and activation of p38 signaling were identified. Unexpectedly, increased numbers of activated cardiac fibroblasts were identified in Bag3 P209L Tg + hearts without increased fibrosis. Together, these findings point to a previously undescribed therapeutic target that may have application to mutation-induced myofibrillar myopathies as well as other common causes of heart failure that commonly harbor misfolded proteins. (Am J Pathol 2016, 186: 1989-2007; http://dx.doi.org/10.1016/j.ajpath.2016.03.017)
\end{abstract}

The Bcl2-associated athanogene (BAG) 3 protein is a member of the BAG family of cochaperones found primarily in skeletal and cardiac muscle. Within muscle cells, BAG3 colocalizes with desmin and $\alpha$-actinin at the sarcomere Z-disk, a primary node where mechanical stimulation is translated to signal transduction pathways allowing the cell to respond to stress. Native BAG3 has been implicated in apoptosis, development, cytoskeletal organization, and autophagy, allowing cells to adapt to external stimuli. ${ }^{1}$ Mechanical stimulation itself induces BAG3 expression, ${ }^{2-4}$ which may indicate its role in the maintenance of the skeletal muscle sarcomere in disease.

Supported by the Jefferson-Pilot Corporation Fellowship in Academic Medicine (M.S.W.), the NIH National Heart, Lung, and Blood Institute (R01HL104129), and the Leducq Foundation (M.S.W.).

Disclosures: None declared. 
Myofibrillar myopathies are a heterogeneous group of skeletal and cardiac muscle diseases caused by mutations in eight sarcomere proteins, including BAG3. ${ }^{5}$ At least 10 mutations have been reported in BAG3, nine resulting in a dilated cardiomyopathy. ${ }^{6-8}$ One of the most commonly reported mutations is the P209L missense mutation, resulting in particularly affected cardiac phenotype, constituting a subset of myofibrillar myopathies leading to both skeletal muscle and cardiac complications. ${ }^{6,9-11}$ Because the P209L mutation is found at amino acid 209, it is worth mentioning that recent studies have found that the Bag3 Ile-Pro-Val (IPV) motif found at amino acids 200 to 213 in Bag3 bind to the heat shock protein HSPB6. ${ }^{12}$ In patient biopsies, the Bag3 P209L mutant leads to increased levels of HSP27 (HSPB1), CRYAB, and Bcl-2 by immunohistochemistry. ${ }^{10}$ The mutant BAG3 complex migrates farther than the wildtype BAG3 complex in denaturing gel electrophoresis, suggesting that the P209L mutant Bag3 interacts less with partner proteins. ${ }^{10}$

Although little is known about the Bag3 P209L mutation, it is distinctly not a loss-of-function mutation. Neonatal cardiomyocytes transfected with Bag3 P209L have a greater susceptibility to apoptosis when stressed, ${ }^{6}$ consistent with $\mathrm{Bag} 3^{-/-}$mice that have this same pro-apoptotic phenotype. ${ }^{11}$ Reducing BAG3 expression in zebrafish results in a severe cardiac defect, ${ }^{8}$ likely related to its structural role facilitating Hsc70 and CapZ, a protein linking actin filaments to the Z-disk. ${ }^{2}$ In zebrafish, expression of Bag3 P209L induces protein aggregates and gradually reduce the pool of BAG3. ${ }^{13}$ However, the underlying pathological mechanisms with which the Bag3 P209L mutation causes disease have not been identified, nor has it been previously associated with autophagy regulation. The slowly progressive loss of cardiac function, a key feature in BAG3 P209L patients, has similarly not previously been identified in animal models.

To elucidate the underlying mechanisms of disease and identify potentially targetable intermediates driving the Bag3 P209L pathology in patients, we generated cardiomyocytespecific ( $\alpha$ MHC-) Bag3 P209L transgenic mice and characterized their progressive cardiac phenotype in vivo over time. Given the increasing appreciation of the role of misfolded proteins in neurodegenerative diseases in heart failure, ${ }^{14}$ we focused on characterizing the misfolded proteins in the Bag3 P209L Tg ${ }^{+}$hearts, and their effects on altering mitochondrial dynamics, effects on native BAG3 expression, and activation of mitogen-activated protein kinase signaling paralleling that found in the pathogenesis of neurodegenerative disease. Unexpected proliferation of cardiac fibroblasts (lacking Bag3 P209L only found in cardiomyocytes) and activation of p38 were identified at 1 year of age, where a progressive systolic and diastolic heart failure was identified by echocardiography. Together, these findings point to a previously undescribed therapeutic target that may have application to mutation-induced myopathies and other common causes of heart failure that harbor misfolded proteins, such as idiopathic dilated cardiomyopathy. ${ }^{15-18}$

\section{Materials and Methods}

\section{Generation of Bag3 P209L Mouse Strain and Experimental Design}

The human Bag3 cDNA with P209L (http://www.ncbi.nlm. nih.gov; GenBank accession number NM_004281) was PCR amplified by the following primers: forward: XhoIATG-Flag-Bag3 (5'-GGCTCGAGATGGACTACAAAGACGATGACGACAAGGGTAGCGCCGCCACCCACT(G-3') and reverse: HindIII-Bag3 with stop codon $\left(5^{\prime}\right.$-CCATTGGGTCGTCGTGGCATCTTCGAAGG- $\left.3^{\prime}\right)$ and cloned into the SalI and HindIII site of the $\alpha \mathrm{MHC}$ promoterpBSIISK $^{+}$construct (gifted by Dr. Jeff Robbins, Cincinnati Children's Hospital, Cincinnati, OH). Positive clones were digested with NotI, generating a 9-kb fragment, which was injected into the pronuclei of BDF1 embryos and implanted into pseudo-pregnant recipient females. Transgene-positive founders were then bred onto C57BL/6 mice (Charles River Laboratories, Inc., Wilmington, MA). Mice were genotyped from isolated tail DNA (DNeasy Tissue Kit; Qiagen Inc., Valencia, CA) at both weaning and post-mortem. The Bag3 transgene was identified using standard PCR conditions (forward primer: 5'-CATGCCAGAAACCACTCAGC- $3^{\prime}$; reverse primer: 5'-GTAGACAGGTCCCACGTAGC-3' ${ }^{\prime}$ or forward primer/F2: $5^{\prime}$-AGAGACGGTGTCAGGAAGGT-3'; reverse primer/R2: 5'-GAACTACCCACCGTAGGGA-3'). The Bag3 transgene expression levels were approximately twofold wild-type levels. All experiments were approved by the Institutional Animal Care and Use Committee review boards at the University of North Carolina and were performed in accordance with federal guidelines.

\section{Experimental Design}

All experiments described used age-matched sibling mice (approximately $50 \%$ male/approximately $50 \%$ female). Conscious echocardiographic analysis of cardiac function began at 4 months of age and repeated until cardiac dysfunction was identified (8 months) through 12 months of age. At 12 months of life, mice were anesthetized with isoflurane, euthanized with cervical spine dislocation, and heart muscle was flash frozen (stored at $-80^{\circ} \mathrm{C}$ ) or perfused by gravity. After abdominal aorta transection, cardiac perfusion was performed using a $23 \mathrm{~g}$ butterfly needle in the apex, first perfusing $10 \mathrm{~mL}$ sterile phosphate-buffered saline, followed by $20 \mathrm{~mL} 4 \%$ paraformaldehyde at a constant rate of $1 \mathrm{~mL} /$ minute. Cardiac tissue was then fixed in $4 \%$ paraformaldehyde for exactly 24 hours, followed by storage in $70 \%$ ethanol, and processed for routine paraffinembedded histology (5 $\mathrm{mm}$ thick sections) and stained with hematoxylin and eosin, Mason's trichrome, or fixed in $2 \%$ paraformaldehyde and $2.5 \%$ glutaraldehyde in $0.15 \mathrm{~mol} / \mathrm{L}$ sodium phosphate buffer $(\mathrm{pH}$ 7.4) overnight and then post-fixed with $1 \%$ osmium tetroxide $/ 0.15 \mathrm{~mol} / \mathrm{L}$ sodium phosphate buffer and processed for transmission electron 
microscopy (TEM). Samples were dehydrated with increasing concentrations of ethanol, infiltrated, and embedded in Poly/Bed812 epoxy resin (Polysciences, Inc., Warrington, PA). Sections (1 $\mu \mathrm{m}$ thick) were prepared to select representative areas by light microscopy, and sections (70 nm ultrathin) were cut with a diamond knife. Sections were mounted on 200 mesh copper grids and stained with $4 \%$ aqueous uranyl acetate and Reynolds' lead citrate.

\section{Quantitative Measure of Mitochondria by qPCR Analysis}

Mitochondrial number was quantified by real-time quantitative PCR (qPCR), and DNA was isolated from $50 \mu \mathrm{L}$ whole-heart homogenates using the DNAeasy Blood and Tissue Kit (Qiagen; catalogue number 69506). Isolated DNA and oligomer primers for mitochondrial cytochrome c oxidase subunit 1 (CO1; mt-CO1), cytochrome b (Cyt-b; mt-Cyb), and NADH dehydrogenase 1 (ND1; mt-nd1) DNA normalized to nuclear H19 (imprinted maternally expressed transcript, nonprotein coding) DNA were run in SYBR Green mastermix by qPCR, including melting curves as previously detailed. ${ }^{19}$

\section{Mouse Echocardiography}

Conscious cardiac transthoracic echocardiography was performed on mice at monthly time points using a VisualSonics Vevo 2100 ultrasound biomicroscopy system (VisualSonics, Inc., Toronto, ON, Canada), as previously described. ${ }^{20}$ Briefly, two-dimensional M-mode echocardiography was performed in the parasternal long-axis view at the level of the papillary muscle on loosely restrained mice by investigators (M.T.Q., M.S.W.) blinded to mouse genotype throughout the process of collection and analysis. Anterior and posterior wall thickness was measured as distance from epicardial to endocardial leading edges. Left ventricular internal diameters were also measured. Left ventricular systolic function was assessed by ejection fraction, left ventrical (LV) ejection fraction \% $=[(\mathrm{LV}$ Volume; diastole-LV Volume; systole/LV Vol; diastole) $\times$ 100], and fractional shortening $\%=[(\mathrm{LV}$ end-diastolic dimension - LV end-systolic diameter)/LV end-diastolic diameter] $\times 100$.

Measurements represent the average of three cardiac cycles from each mouse. To measure diastolic function, Doppler measurement of the mitral valve flow was taken in an apical view into the LV outflow tract under isoflurane anesthesia, as previously described. ${ }^{21}$

\section{Measurement of Autophagic Flux}

Autophagic flux was determined in vivo from the titration of bafilomycin A1 to optimize animal survival without eliciting other major dysfunction, while inhibiting autophagy in the heart, as previously described. ${ }^{22,23}$ Briefly, stock bafilomycin A1 (number 201550A; Santa Cruz Biotechnology, Dallas,
$\mathrm{TX} ; 3 \mathrm{mmol} / \mathrm{L}$ stock in dimethyl sulfoxide) diluted in phosphate-buffered saline ( $40 \%$ dimethyl sulfoxide final) was injected i.p. to a final concentration of 3 micromol per kilogram in $200 \mu \mathrm{L}$ and repeated 1 hour later. One hour after the second injection, mice were euthanized and heart tissues were collected for analysis by Western immunoblot for LC3B isoforms (Sigma-Aldrich number L7543).

\section{Histological and TEM Morphometric Analysis}

\section{Cross-Sectional Areas}

Imaging of hematoxylin and eosin- and Masson's trichromestained sections was obtained using Aperio Scanscope and Aperio Imagescope software version 10.0.36.1805 (Aperio Technologies, Inc., Vista, CA). Masson's trichrome-stained images were taken using Aperio Imagescope (TIFF) and analyzed using NIH ImageJ software version 1.47 (NIH, Bethesda, MD) using Aperio exported image ruler. Cardiomyocyte cross-sectional area was measured using ImageJ. A minimum of 100 random cardiomyocytes was imaged from at least three different sections from four biological replicates per experimental group.

\section{Fibrosis Analysis}

Using ImageScope, positive pixel countv9 algorithm was used on Masson's trichrome-stained histological section (hue value, 0.66; hue width, 0.1) analyzing tissue sections isolated using the pen tool. After analysis, the calculated $\mathrm{N}$ positive/ $\mathrm{N}$ total was multiplied by 100 to give a percentage collagen content (identifying the blue as a percentage of the tissue present).

\section{Nuclei Counts}

The ImageScope positive pixel countv9 algorithm was used to analyze hematoxylin and eosin-stained slides for nuclei (hue value, 0.1; hue width, 0.45; color saturation 0.3), similarly isolated for fibrosis. The nuclei per area was identified per $\mathrm{mm}$ squared.

\section{TEM Morphometric Analysis}

Cardiac sections were observed with a LEO EM910 TEM operating at $80 \mathrm{kV}$ (LEO Electron Microscopy, Thornwood, NY) and photographed with a Gatan Orius SC1000 CCD Digital Camera and Digital Micrograph 3.11.0 (Gatan, Pleasanton, CA). Mitochondria cross-sectional areas were measured using NIH ImageJ at magnifications of $\times 5000$ to $\times 10,000$. The global scale was set according to the imagespecific scale generated by Gatan camera output. An average of 2500 mitochondria were analyzed from 30 fields from multiple levels from three hearts per mouse cohort.

Immunofluorescence Staining of Cardiac Histological Sections for Vimentin, AIF, FLAG (Bag3 P209L), and TUNEL

Immunostaining was performed as described previously. ${ }^{24,25}$ Cardiac sections were stained with antibodies against the 
following: i) vimentin (1:100, catalogue number SC-6260; Santa Cruz Biotechnology, Dallas, TX), or an unrelated isotype mouse, rabbit, or goat IgG (as a negative control) at $4{ }^{\circ} \mathrm{C}$ overnight; ii) apoptosis-inducing factor (AIF; 1:250; Abcam, Cambridge, MA) or an unrelated isotype mouse, rabbit, or goat IgG (as a negative control) at $4^{\circ} \mathrm{C}$ overnight; iii) FLAG (DYKDDDK Tag) (1:100; Cell Signaling Technology, Danvers, MA) or an unrelated isotype mouse, rabbit, or goat IgG (as a negative control) at $4{ }^{\circ} \mathrm{C}$ overnight. Slides were then incubated with Alexa Fluor 488-conjugated secondary antibodies and counterstained with DAPI (Vector Laboratories, Burlingame, CA). Sections were then treated with Alexa Fluor 488-conjugated secondary antibodies and counterstained with DAPI. Total positive vimentin stain cells were identified and counted in each of the four cross-sectional defined areas using Meta-Morph software version 7.8 (Molecular Devices). Images were taken using EVOS XL Core cell imaging system (Life Technologies).

Apoptosis was determined in histological sections by identifying the presence of fragmented DNA by terminal deoxynucleotidyl transferase dUTP nick end labeling (TUNEL) using the Roche TUNEL in situ staining kit (Roche Molecular Biochemicals, Basel, Switzerland), according to the manufacturer's instructions. To detect DNA fragmentation associated with apoptosis, we used a fluorescence-based TUNEL followed by counterstaining with DAPI. Histological sections were treated with a recombinant DNase I to allow TUNEL labeling of all nuclei, which were used as positive controls.

\section{Western Blot Analysis}

For Western blot analysis, 24- to 36-day-old hearts were homogenized in radioimmunoprecipitation assay buffer and loaded $10 \mu \mathrm{g} / \mathrm{lane}$ and blotted for using mouse anti-FLAG M2 (Sigma number A8592, 1:1000) to detect the Flagtagged Bag3 P209L protein, anti-serum raised against purified GST-BAG3 recognizing both human/mouse BAG3, and anti-actin (Calbiochem Corp., catalogue number CP01, Ab-1/JLA20). Western analysis of ventricular tissue was performed on lysates generated from approximately $25 \mathrm{mg}$ tissue placed in $8 \mathrm{~mol} / \mathrm{L}$ Urea Lysis Buffer $(8 \mathrm{~mol} / \mathrm{L}$ Urea Sigma, catalogue number U0631, $5 \mathrm{~mol} / \mathrm{L} \mathrm{NaH}_{2} \mathrm{PO}_{4} \mathrm{Sigma}$, catalogue number $\mathrm{S} 3139,1 \mathrm{~mol} / \mathrm{L}$ Tris-Cl, $\mathrm{pH}$ 8.0) at a ratio of $15 \mu \mathrm{L}$ lysis buffer/mg of tissue and was homogenized at $4^{\circ} \mathrm{C}$ (TissueLyser LT, Qiagen, catalogue number 85600 ) for 2 minutes. The homogenate was centrifuged at $4{ }^{\circ} \mathrm{C}$, $16,000 \times g$ for 15 minutes, and the supernatant stored at $-80^{\circ} \mathrm{C}$. Protein concentration was determined using the Bio-Rad DC Protein Assay Reagent Package (Bio-Rad Laboratories, Inc., Hercules, CA, catalogue number 5000116). Proteins (30 to $50 \mu \mathrm{g} / \mathrm{lane}$ ) were resolved on NuPAGE Bis-Tris or Tris-Acetate 10- and 15-well gels. Rabbit anti-ubiquitin (Sigma L7543, 1:500) was used to measure changes in ubiquitination. Mouse anti-NF- $\kappa \mathrm{B}$ p65 (total) and rabbit anti-phospho-NF-кB p65 (Ser536) were used to measure NF- $\kappa \mathrm{B}$ signaling (Cell Signaling Technologies, catalogue number 4767, each 1:500). Rabbit antiLC3 (Sigma L7543, 1:500) was used to measure autophagic flux. Rabbit anti-p38 and phospho-p38 (Cell Signaling, 9212, 9211, each 1:1000) was used to measure p38 activation. Rabbit anti-PRDX1 and anti-SSADH (Sigma SAB2101877 and PRS4807, each 1:1000) were used to detect periredoxin and succinic semialdehyde dehydrogenase. Primary antibodies were diluted in $1 \times$ Tris-buffered saline and Tween 20 or $4 \%$ bovine serum albumin and incubated at $4{ }^{\circ} \mathrm{C}$ overnight. Horseradish peroxidaselabeled secondary antibodies against mouse (Sigma A9917, 1:10,000), goat (Sigma A4174, 1:10,000), and rabbit (Sigma A9169, 1:5000) were used to detect the primary antibodies diluted in $1 \times$ Tris-buffered saline and Tween 20 and incubated 1 hour at room temperature. Mouse anti- $\beta$-actin (Sigma, Inc., catalogue number A2228, 1:4000) or mouse anti-glyceraldehyde-3-phosphate dehydrogenase (Sigma, Inc., catalogue numberG8795, 1:5000) were used as a loading control. Secondary antibody horseradish peroxidase was detected using ECL Select (GE Healthcare, catalogue number RPN2235) and imaged using the MultiDoc-it Imaging System (UVP, LLC, Upland, CA).

\section{RNA Isolation and Quantitative PCR Analysis of Gene Expression, Mitochondrial Dynamics}

Total RNA was isolated using TRIzol reagent, according to the manufacturer's protocols (Life Technologies, Inc., catalogue number 15596-026). Approximately $25 \mathrm{mg}$ of cardiac ventricular tissue was put into TRIzol reagent and homogenized at $4^{\circ} \mathrm{C}$ (TissueLyser LT, Qiagen, catalogue number 85600). Total mRNA expression was determined using a two-step reaction. cDNA was made from total RNA using the iScript Reverse Transcription Supermix for RT-qPCR kit (catalogue number $170-8841$, Bio-Rad), with a total volume of $20 \mu \mathrm{L}$ per reaction. The complete reaction mix was incubated in an Eppendorf Cycler (Hamburg, Germany) using the following protocol: priming 5 minutes at $25^{\circ} \mathrm{C}$, reverse transcription 30 minutes at $42^{\circ} \mathrm{C}$, room temperature inactivation 5 minutes at $85^{\circ} \mathrm{C}$. PCR products were amplified on a Roche Lightcycler 480II system using cDNA, Taqman Probes (Applied Biosciences), and Lightcycler 480 Probe Master Mix 2X (catalogue number 04707494 001). The TaqMan probes used in this study were Hs99999901_s1 (18S), Mm00600555_m1 ( $\beta$ MHC, Myh7), Mm01255747_g1 (ANF), Mm00435304_g1 (BNP), Mm00808218_g1 (Acta1, skeletal muscle $\alpha 1$-actin), Mm01278171_m1 (mouse Bag3, spanning exons 2 to 3), Hs00188713_m1 (human Bag3, spanning exons 1 to 2), Opa1 (Mm00453879_m1), Drp1 (Mm01342903_m1), Fis1 (Mm00481580_m1), and Mfn1 (Mm00612599_m1) (Applied Biosystems, Inc., Foster City, CA). Samples were run in triplicate, and relative mRNA expression was determined using $18 \mathrm{~S}$ as an internal endogenous control. RNase-free water, 2X Master Mix, Taqman Probe, or Roche UPL primer and probe, and cDNA were used for each reaction. 
Protein Aggregation Assay and Preamyloid Oligomer Staining

Protein aggregation was assessed using an Enzo Proteostat Protein Aggregation Assay (ENZ-51023, Farmingdale, NY) in conjunction with their preformulated aggregation standards (ENZ-51039). Briefly, protein lysates were prepared from flash-frozen apical cardiac tissues using $8 \mathrm{~mol} / \mathrm{L}$ urea lysis buffer, protein concentrations were determined by Bradford assays, and assays were performed in 96-well, black-walled, clear-bottom plates read on a Clariostar microplate reader at 550-nm excitation and $600-\mathrm{nm}$ emission with gain setting between 1000 and 2000. Preamyloid oligomer staining was performed as previously described using the A11 and MF20 antibodies. ${ }^{26}$

\section{Nontargeted Metabolomics Determination by Gas Chromatography-Mass Spectrometry Instrumentation}

Cardiac tissue was flash frozen with liquid nitrogen cooled in a biopress, a fraction weighed (approximately 25 to 30 $\mathrm{mg}$ weight), finely ground, and added to fresh $50 \%$ acetylnitrile, $50 \%$ water, and $0.3 \%$ formic acid at a standard concentration of $25 \mathrm{mg} / 475 \mathrm{mcl}$ buffer, then fully homogenized on ice for 10 to 25 seconds and placed on dry ice/ stored at $-80^{\circ} \mathrm{C}$. Samples were crash deprotonized by methanol precipitation and spiked with D27-deuterated myristic acid (D27-C14:0) as an internal standard for retention-time locking and dried. The trimethylsilyl (TMS)D27-C14:0 standard retention time was set at $* 16.727 \mathrm{mi}-$ nutes. Reactive carbonyls were stabilized at $50^{\circ} \mathrm{C}$ with methoxyamine hydrochloride in dry pyridine. Metabolites were made volatile with TMS groups using N-methyl-N(trimethylsilyl) trifluoroacetamide or MSTFA with catalytic trimethylchlorosilane at $50^{\circ} \mathrm{C}$. Gas chromatography-mass spectrometry methods were adopted from Roessner et $\mathrm{al}^{27}$ Fiehn et $\mathrm{al}^{28}$ and Kind et $\mathrm{al},{ }^{29}$ which used a $6890 \mathrm{~N}$ gas chromatograph connected to a 5975 Inert single quadrupole mass spectrometer (Agilent Technologies, Santa Clara, CA). The two wall-coated, open-tubular gas chromatography columns connected in series are both from J\&W/Agilent (part 122-5512), DB5-MS, $15 \mathrm{~m}$ in length, $0.25 \mathrm{~mm}$ in diameter, with an $0.25-\mathrm{lm}$ luminal film. Positive ions generated with conventional electron-ionization (EI) at 70 $\mathrm{eV}$ are scanned broadly from 600 to $50 \mathrm{~m} / \mathrm{z}$ in the detector throughout the 45-minute cycle time. Data were acquired and analyzed as previously described. ${ }^{30,31}$ All raw data and transformed data included in the analysis are included in Supplemental Tables S1 and S2, respectively.

\section{Thioredoxin Reductase Assay}

Flash-frozen cardiac tissue (approximately $20 \mathrm{mg}$ ) was homogenized at a ratio of $10 \mu \mathrm{L}$ lysis buffer $/ \mathrm{mg}$ of tissue according to the manufacturer's protocols to prepare assay homogenate (TissueLyser LT, Qiagen, catalogue number
85600). Protein concentration was determined using the Pierce $660 \mathrm{~nm}$ Protein Assay Reagent (Thermo Fisher Scientific, catalogue number 22660) and assayed for thioredoxin reductase activity using a Thioredoxin Reductase Assay (Sigma, catalogue number CS0170) according to the manufacturer's protocol. Protein $(100 \mu \mathrm{g})$ was incubated on a U bottom 96-well plate with DTNB containing reaction mix in the presence or absence of a thioredoxin reductase-specific inhibitor and read at $412 \mathrm{~nm}$ using a Clariostar microplate reader.

\section{Statistical Analysis}

Prism 6 for Mac OS X (Version 6.0f, August 5, 2014) or SigmaPlot for Windows (Version 11.0 Build 11.0.0.77) was used to plot and statistically analyze data using a $t$-test with significance defined as $P<0.05$. Statistical analysis on metabolomics data was performed as previously described. ${ }^{30,31}$ Briefly, Metaboanalyst (v2.0) run on the statistical package R (v2.14.0) used metabolite peak areas (as representative of concentration). ${ }^{32,33}$ These data were first analyzed by an unsupervised principal component analysis. To sharpen the separation between groups, data were next analyzed using a partial least squares discriminant analysis to further determine which metabolites were responsible for separating these two groups. The specific metabolites contributing most significantly to the differences identified by partial least squares discriminant analysis between Bag3 P209L and wild-type control group hearts were determined using the variable importance in projection (VIP) analysis in the Metaboanalyst environment. The metabolites that best differentiated the groups were then individually tested using the $t$-test (Microsoft Excel 2011, Seattle, WA). The VIP and $t$-test significant metabolites were matched to metabolomics pathways using the Pathway Analysis feature in Metaboanalyst 2.0. Heat maps of the metabolite data (individual and grouped) were generated using the GENE E software version 3.0.2014 (http://www.broadinstitute.org/cancer) software/GENE-E/index.html; last accessed February 20, 2016).

\section{Results}

The BAG3 P209L missense mutation in exon 3 is one of the most recently identified causes of myofibrillar myopathy resulting in cardiac complications. ${ }^{10}$ In contrast to other myofibrillar myopathies, the BAG3 cardiomyopathy manifests as hypertrophic cardiomyopathy in the first to second decades of life. ${ }^{5,10} \mathrm{Bag} 3$ constitutive expression is restricted to few cell types, including skeletal muscle and cardiomyocytes. ${ }^{2}$ However, it is induced in a subset of cells (retinal, glial, epithelial, leukocytes) by a variety of stressors to induce resistance. ${ }^{34-37}$ To generate a cardiac model that paralleled human disease without the indirect effects of a skeletal phenotype, including respiratory distress and 


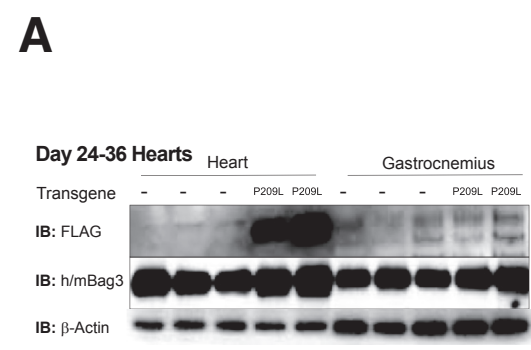

D
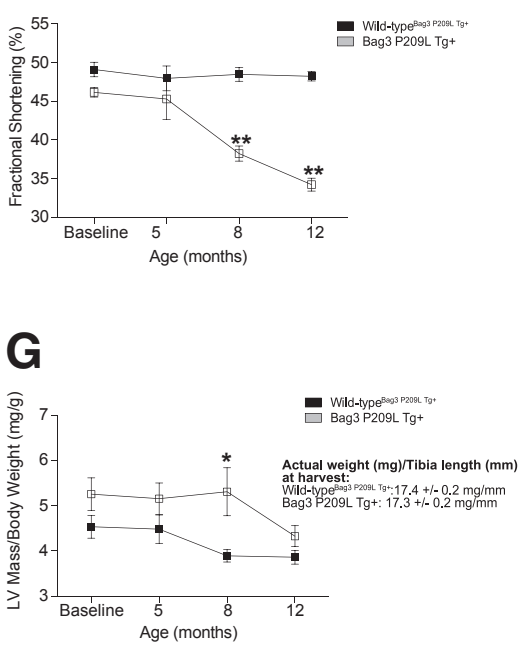

B Mendelian Ratios: Bag3 P209L Tg+ $x$ Wild-type Not significantly different from
expected ratios (Table 1) by bino

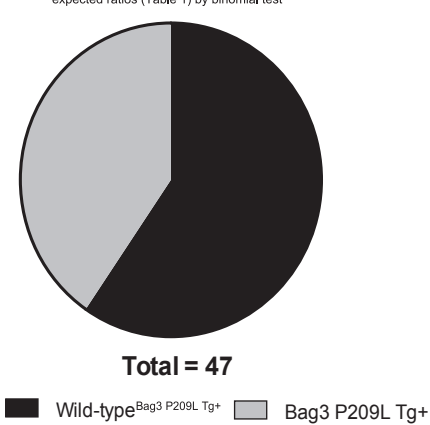

E

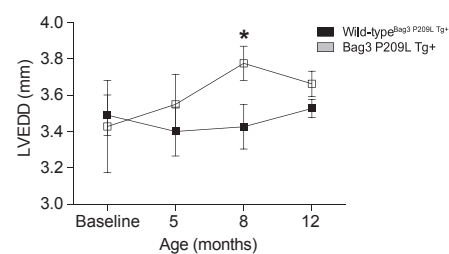

H Harvested Heart Weight at 12 Months

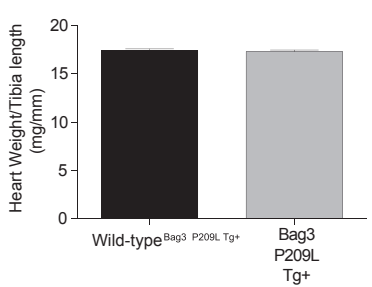

C

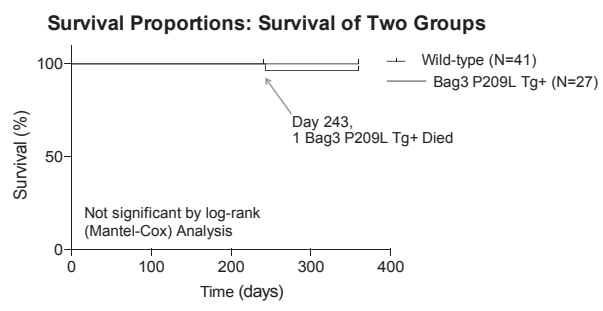

$\mathbf{F}$

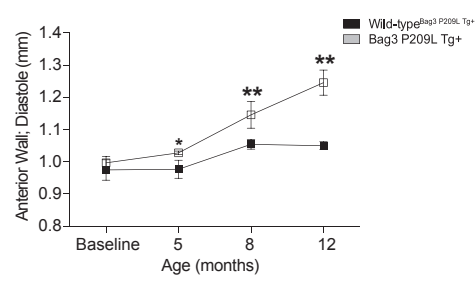

Diastolic Function by Doppler Analysis
of Mitral Valve (E/a Ratio) at 12 Months

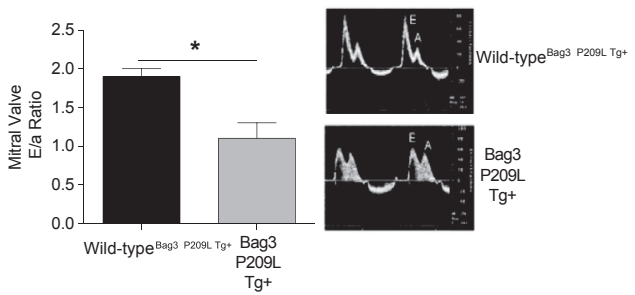

Figure 1 Cardiac function and natural history of aMHC-Bag3 P209L transgenic mouse cardiac phenotype up to 12 months of age. A: Western blot analysis of Bag3 P209L expression by anti-FLAG (FLAG-Bag3 P209L) demonstrating specificity and by anti-BAG3 (human and mouse) to compare relative levels of total BAG3 in cardiac and gastrocnemius tissue from $\alpha \mathrm{MHC}-\mathrm{Bag} 3 \mathrm{P} 209 \mathrm{~L}$ and littermate wild-type mice. B: Mendelian ratios of offspring at the time of genotyping at 14 to 21 days post-partum. C: Kaplan-Meier survival curves of Bag3 P209L Tg ${ }^{+}$and sibling wild-type mice. D-G: Temporal changes in fractional shortening \%, calculated as (LVEDD-LVESD)/LVEDD $\times 100$, see Table 2 (D), left ventricular end diameter; diameter (E), anterior wall thickness in diastole (AWTD) (F), and left ventricular mass (G), corresponding to the complementary morphometrics in Table 2. H: Measured total heart weight (normalized to tibia length) at 1 year of age. I: Analysis of diastolic function made from Doppler measures of mitral valve at 1 year of age. A $t$-test was used to compare genotypes at each time point. Data are expressed as means \pm SEM $(\mathbf{D}-\mathbf{I}) . N=47(\mathbf{B}) ; N=68(\mathbf{C}) ; N=3(\mathbf{D}-\mathbf{G}$, baseline Bag3 P209LTg + and 5 months Bag3 P209LTg +$) ; N=4(\mathbf{D}-\mathbf{G}, 8$

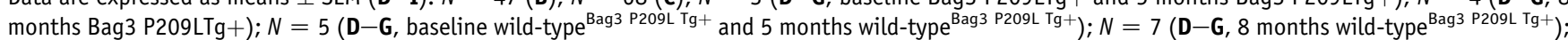
$N=10\left(\mathbf{D}-\mathbf{G}, 12\right.$ months wild-type ${ }^{\text {Bag3 P209L Tg+ }}$ and BAG3 P209LTg +$) ; N=4(\mathbf{H}, \mathrm{Bag} 3 \mathrm{P} 209 \mathrm{LTg}+) ; N=7$ (H, wild-type $\left.{ }^{\mathrm{Bag} 3 \mathrm{P} 209 \mathrm{~L} \mathrm{Tg}+}\right) ; N=3$ per group $(\mathbf{I})$. ${ }^{*} P<0.05,{ }^{* *} P<0.005$ versus age-matched sibling wild-type mice. IB, immunoblot; LV, left ventricle; LVEDD, left ventricular end-diastolic diameter.

limited mobility, we generated an $\alpha$ MHC-driven BAG3 P209L transgenic mouse. The resulting mouse exhibited a modest constitutive increase in total BAG3 expression (approximately twofold wild-type levels) (Figure 1A), localized to the heart (cardiomyocyte). Because we crossed Bag3 P209L $\mathrm{Tg}^{+}$with wild-type mice, we looked for evidence of prenatal death by comparing the expected $50 \%$ ratios with the observed. There was no evidence that the Bag3 P209L $\mathrm{Tg}^{+}$offspring were underrepresented, with $>50 \%$ Bag3 P209 $\mathrm{L} \mathrm{Tg}^{+}$by genotyping (Figure $1 \mathrm{~B}$ and Table 1). The distribution of offspring with Bag3 P209L $\mathrm{Tg}^{+}$and sex similarly did not differ between groups (Supplemental Figure S1 and Supplemental Table S3), demonstrating that the Bag3 P209 $\mathrm{Tg}^{+}$did not alter viability during development. Body weight from 4 months of age through 12 months was not significantly different between wild-type Bag3 P209L $\mathrm{Tg}^{+}$mice, as shown in Table 2 (row 1).

Because the Bag3 P209L $\mathrm{Tg}^{+}$was expressed specifically in cardiomyocytes, we focused our analysis on cardiac function, performing conscious echocardiography over time, starting at 4 months of age and continuing through 1 year, along with mechanistic studies. Postnatally, we identified only one mouse that died prematurely, which was a Bag3 P209 $\mathrm{L} \mathrm{Tg}^{+}$mouse on day 243 (Figure 1C). Unfortunately, we were unable to perform a necropsy to determine any contributions of the mutation to its death, but did confirm we had identified systolic dysfunction by echocardiography 
Table 1 Analysis of Mendelian Ratios Breeding Bag3 P209L Tg ${ }^{+}$with Strain-Matched Wild-type ${ }^{\text {Bag3 P209L Tg+ }}{ }^{\text {Mice }}\left(50 \%\right.$ Expected Tg ${ }^{+}$)

\begin{tabular}{lllccc}
\hline Outcome & Expected (no.) & Observed (no.) & Expected (\%) & Observed (\%) & $95 \%$ CI of observed \% \\
\hline Wild-type Bag3 P209L Tg+ $^{\text {B }}$ & 23.5 & 28 & 50 & 59.57 & $44.27-73.63$ \\
Bag3 P209L Tg+ & 23.5 & 19 & 50 & 40.43 & $26.37-55.73$ \\
Total & 47 & 47 & 100 & 100 & \\
\hline
\end{tabular}

A binomial test was performed and a one-tailed and two-tailed $P$ was determined to be not significant $(0.1215$ and 0.2430 , respectively). See Figure $1 \mathrm{~B}$ for pie chart of distribution.

prior. By 8 months of age, significant systolic dysfunction was seen, evidenced by decreases in fractional shortening (Figure 1D) and ejection fraction (Table 2). Although Bag3 P209L $\mathrm{Tg}^{+}$hearts demonstrated significant dilation at 8 months (Figure 1E) and had significantly increased anterior wall thickness (Figure 1F), there was no difference in LV mass determined by echocardiographic measurements (Figure 1G) or measured heart weight at 12 months of age (Figure $1 \mathrm{H}$ ).

Of the six genes found to be mutated and leading to myofibrillar myopathies [desmin, aB-crystallin, myotilin, LDB3 (ZASP), FLNC, and BAG3], only $15 \%$ to $30 \%$ of patients with these mutations present with a cardiomyopathy. ${ }^{5}$ As the disease progresses, up to $60 \%$ develop cardiomyopathies, including dilation (17\%), restrictions (12\%), and/or hypertrophic (6\%) cardiomyopathy. ${ }^{38}$ The first case of a Bag3 P209L mutation reported in 2009 identified a childhood onset with severe progressive muscle weakness with atrophy, along with a restrictive cardiomyopathy pattern. $^{5,10}$ Because of the restrictive cardiomyopathy pattern, we hypothesized that the Bag3 P209L $\mathrm{Tg}^{+}$mice had exhibited diastolic dysfunction, being unable to relax. We performed Doppler analysis on the mitral valve in Bag3 P209 $\mathrm{L} \mathrm{Tg}^{+}$mice at 12 months of age and determined an E/a waveform ratio (Figure 1I). Wild-type mice exhibited a dynamic relation during the cardiac cycle, exhibited an E/a ratio of nearly 2.0 , consistent with healthy human heart function generally around 1.5 to $2.0 \mathrm{E} / \mathrm{a}$ ratio. In contrast, the Bag3 P209L $\mathrm{Tg}^{+}$hearts had a reduced diastolic function, with an E/a ratio of approximately 1.0 (Figure 1I).

The severity of the heart failure was limited in the Bag3 P209 $\mathrm{L} \mathrm{Tg}^{+}$hearts, because there was no evidence of congestive heart failure, such as fluid accumulation in the lungs (Figure 2A). Blinded cross-sectional analysis (M.T.Q.) of Masson's trichrome-stained histology revealed that the Bag3 P209L $\mathrm{Tg}^{+}$hearts exhibited a significant increase in cardiomyocyte size (Figure 2B), with no evidence of increased fibrosis, quantified by algorithmic

Table 2 High-Resolution Transthoracic Echocardiography Performed on Conscious Bag3 P209L Tg ${ }^{+}$and Strain-Matched Sibling Controls over 1 Year

\begin{tabular}{|c|c|c|c|c|c|c|c|c|}
\hline Variable & $\begin{array}{l}\text { Baseline wild- } \\
\text { type }{ }^{\text {Bag3 P209L Tg+ }} \\
\text { control }\end{array}$ & $\begin{array}{l}\text { Baseline } \\
\text { Bag3 } \\
\text { P209 } \mathrm{Tg}^{+}\end{array}$ & $\begin{array}{l}5 \text { Months wild- } \\
\text { type }{ }^{\text {Bag3 P209L Tg+ }} \\
\text { control }\end{array}$ & $\begin{array}{l}5 \text { Months } \\
\text { Bag3 } \\
\text { P209 } \mathrm{Lg}^{+}\end{array}$ & $\begin{array}{l}8 \text { Months wild- } \\
\text { type }{ }^{\text {Bag3 P209L Tg+ }} \\
\text { control }\end{array}$ & $\begin{array}{l}8 \text { Months } \\
\text { Bag3 } \\
\text { P209L Tg }\end{array}$ & $\begin{array}{l}12 \text { Months wild- } \\
\text { type }{ }^{\text {Bag3 P209L Tg+ }} \\
\text { control }\end{array}$ & $\begin{array}{l}12 \text { Months } \\
\text { Bag3 } \\
\text { P209L Tg }\end{array}$ \\
\hline$N$ & 5 & 3 & 5 & 3 & 7 & 4 & 10 & 10 \\
\hline AWTS (mm) & $1.74 \pm 0.05$ & $1.68 \pm 0.03$ & $1.77 \pm 0.05$ & $1.73 \pm 0.06$ & $1.82 \pm 0.04$ & $1.64 \pm 0.05^{*}$ & $1.81 \pm 0.04$ & $1.74 \pm 0.04$ \\
\hline LVESD (mm) & $1.83 \pm 0.06$ & $1.85 \pm 0.15$ & $1.9 \pm 0.11$ & $1.87 \pm 0.16$ & $1.76 \pm 0.06$ & $2.33 \pm 0.12^{*}$ & $1.83 \pm 0.03$ & $2.28 \pm 0.05^{*}$ \\
\hline PWTD & $0.89 \pm 0.01$ & $0.97 \pm 0.02^{*}$ & $0.96 \pm 0.03$ & $1.03 \pm 0.02$ & $1.06 \pm 0.02$ & $0.99 \pm 0.04$ & $1.06 \pm 0.02$ & $0.99 \pm 0.04^{*}$ \\
\hline LV Mass (mg) & $121.8 \pm 5.0$ & $124.0 \pm 16.1$ & $127.2 \pm 11.3$ & $129.8 \pm 7.3$ & $137.3 \pm 8.2$ & $145.1 \pm 10.2$ & $137.3 \pm 8.1$ & $145.1 \pm 10.2$ \\
\hline LV Vol; d (mL) & $54.4 \pm 4.1$ & $49.2 \pm 8.3$ & $57.0 \pm 6.5$ & $47.7 \pm 4.5$ & $49.0 \pm 4.9$ & $61.8 \pm 8.3$ & $51.9 \pm 1.7$ & $64.8 \pm 2.3^{*}$ \\
\hline LV Vol; s (mL) & $10.2 \pm 0.9$ & $10.7 \pm 2.1$ & $11.4 \pm 1.6$ & $11.0 \pm 2.5$ & $9.3 \pm 0.9$ & $19.0 \pm 2.6^{*}$ & $10.1 \pm 0.4$ & $17.8 \pm 1.1^{*}$ \\
\hline PWTH $^{\dagger}(\%)$ & $63.6 \pm 10.0$ & $44.7 \pm 5.6$ & $53.8 \pm 3.8$ & $39.2 \pm 6.2$ & $55.9 \pm 4.2$ & $52.8 \pm 9.4$ & $59.7 \pm 4.5$ & $33.6 \pm 2.0^{*}$ \\
\hline RWT; $d^{\ddagger}$ & $0.52 \pm 0.02$ & $0.58 \pm 0.04$ & $0.52 \pm 0.03$ & $0.61 \pm 0.03$ & $0.62 \pm 0.03$ & $0.53 \pm 0.04$ & $0.60 \pm 0.01$ & $0.66 \pm 0.02^{*}$ \\
\hline RWT; $s^{\S}$ & $1.75 \pm 0.09$ & $1.69 \pm 0.11$ & $1.73 \pm 0.14$ & $1.72 \pm 0.16$ & $1.98 \pm 0.09$ & $1.35 \pm 0.06^{*}$ & $1.91 \pm 0.04$ & $1.44 \pm 0.04^{*}$ \\
\hline
\end{tabular}

Baseline echos taken at 4 months of age. Approximately $50 \%$ female and approximately 50\% male mice were assayed throughout the study. Data represent means \pm SEM. A $t$-test was performed comparing Bag3 $\mathrm{P} 209 \mathrm{~L} \mathrm{Tg}^{+}$to sibling wild-type controls.

$* P<0.05$.

${ }^{\dagger}$ PWTH $=\left[\left(\right.\right.$ LVPW; $s-$ LVPW; d ${ }^{*} 100 \% /$ LVPW; d $]$.

${ }^{\ddagger}$ RWT; $d=$ LVPW; $d+$ LVAntW; d/LVEDDd.

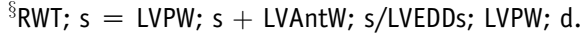

AWTD, anterior wall thickness in diastole; AWTS, anterior wall thickness in systole; bpm, heart beats per minute; $d$, in diastole; EF\%, ejection fraction calculated as (end Simpson's diastolic volume - end Simpson's systolic volume)/end Simpson's diastolic volume * 100; ExLVD, external left ventricular diameter; FS, fractional shortening, calculated as (LVEDD - LVESD)/LVEDD $\times 100 ;$ HR, heart rate; LVEDD, left ventricular end-diastolic dimension; LVESD, left ventricular end-systolic dimension; ND, not determined; PWTD, posterior wall thickness in diastole; PWTH, posterior wall thickness; PWTS, posterior wall thickness in systole; RWT; relative wall thickness; s, in systole; vol, volume. 
A Heart Size Analysis
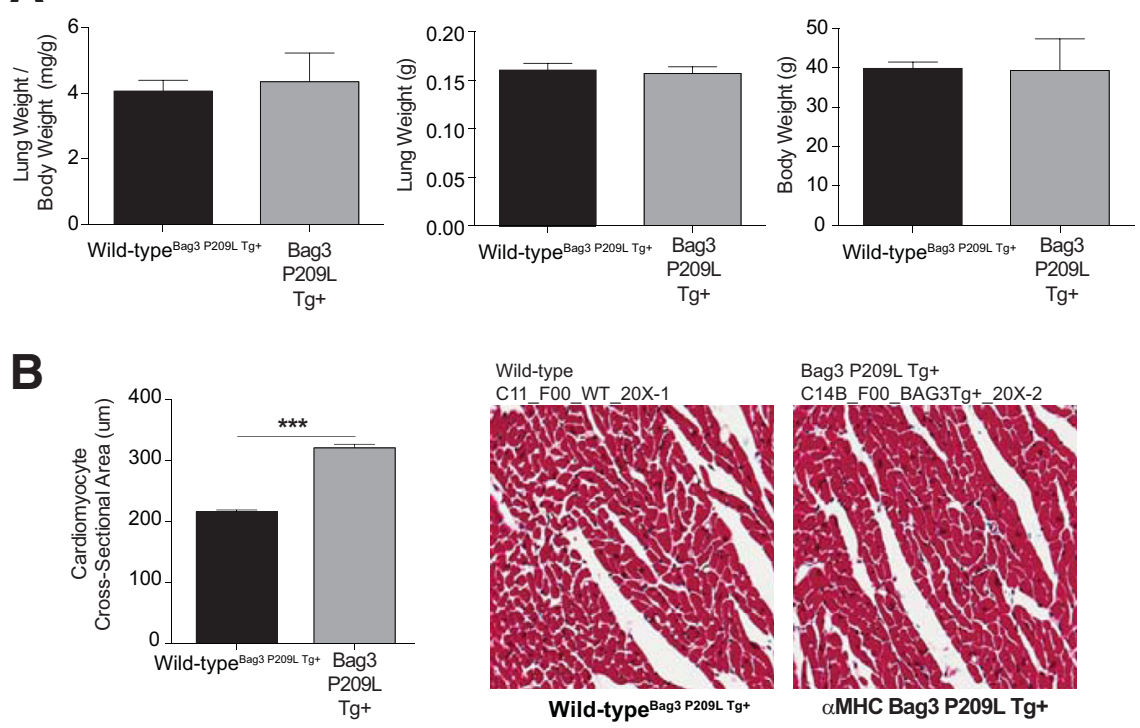

\section{C}

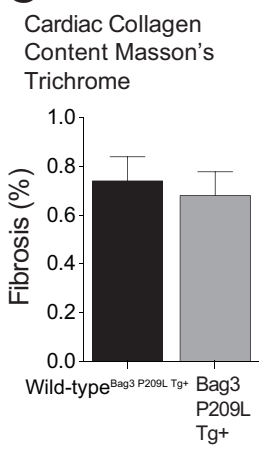

D
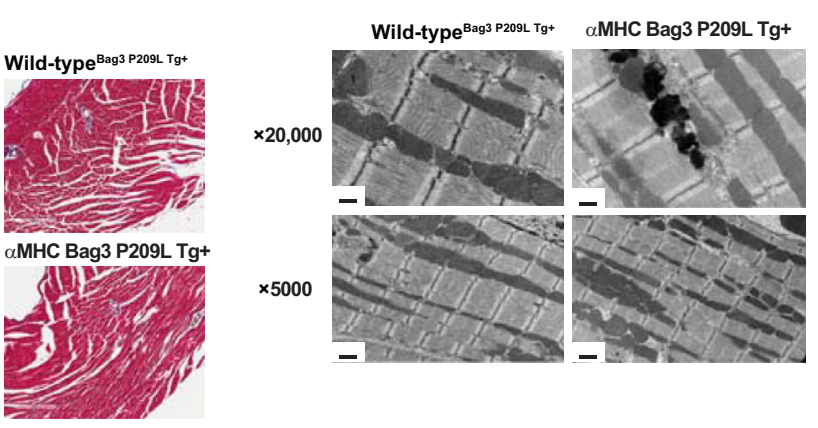

Figure 2 Morphometric analysis of aMHCBag3 P209L transgenic hearts. A: Heart weight and lung weights at 12 months of age. B: Crosssectional analysis of cardiomyocytes from Masson's trichrome-stained histological sections. C: Analysis of collagen in aMHC-Bag3 P209L and littermate wild-type hearts by nonbias computer logarithm analysis of collagen as a measure of fibrosis $\left(N=6\right.$ wild-type ${ }^{\text {Bag3 P209L } \mathrm{Tg}+}, N=4$ Bag3 P209 $\left.\mathrm{Lg}^{+}\right)$. D: Transmission electron microscopy analysis of $\alpha \mathrm{MHC}-\mathrm{Bag} 3 \mathrm{P} 209 \mathrm{~L}$ and littermate wild-type mice at $\times 5$ and $\times 20,000$ $(N=3$ /group). A $t$-test was used to compare genotypes at each time point. Measurements represent means \pm SEM $(\mathbf{A}-\mathbf{C}) . N=3$ per group $(\mathbf{A}-\mathbf{C}) .{ }^{* *} P<0.001$. Scale bars: $0.5 \mu \mathrm{m}(\mathbf{D}$, top row); $1 \mu \mathrm{m}$ (D, bottom row). Original magnification: $\times 20(B) ; \times 10($ C). analysis of collagen (Figure 2C). Transmission electron microscopy revealed no alterations or abnormalities in the Bag3 P209L sarcomere structures throughout the heart (Figure 2D). Taken together, the Bag3 P209L $\mathrm{Tg}^{+}$hearts were normal at birth, and progressively developed both systolic and diastolic heart failure at 1 year of age, with no evidence of prenatal lethality and minimal mortality $(3.7 \%=1 / 27)$.

To better understand the mechanistic cause of the observed cardiac dysfunction, we next investigated how alterations in autophagy, also recognized as the lysosomal degradation pathway, may be related to the cardiac dysfunction seen in the Bag3 P209 $\mathrm{Lg}^{+}$hearts. The primary reason autophagy was of interest in this model was the fact that wild-type Bag3 has recently been implicated in the regulation of chaperone-assisted selective autophagy in muscle. $^{14,39}$ In these studies, the protein Starvin and Bag3 coordinated machinery were required for the maintenance of the $\mathrm{Z}$ disk while Bag3 coordinated the connection between HSC70, the small heat shock protein HspB8, and the ubiquitin ligase CHIP with the autophagic ubiquitin adaptor p62. ${ }^{40}$ Second, mutations in other myofibrillar proteins such as $\alpha \mathrm{B}$ crystallin (eg, CryAB R120G) have been shown to induce endoplasmic reticulum stress ${ }^{41}$ and increase autophagy to enhance the removal of proteotoxic misfolded proteins. $^{42}$ We first investigated if amyloid fibrils were present by measuring amyloid using a dye-based Proteostat assay to measure protein aggregation. In Bag3 P209L hearts expressing the transgenic protein at 12 months of age (Figure 3A), no increase in protein aggregation was detected in heart lysates compared with wild-type ${ }^{\mathrm{Bag} 3 \mathrm{P} 209 \mathrm{~L} \mathrm{Tg}+}$ mice in vivo (Figure $3 \mathrm{~B}$ ). We next investigated if alterations in autophagic flux were present by quantifying the amount of LC3II levels in the heart. No significant differences were seen between Bag3 P209L $\mathrm{Tg}^{+}$and age-matched wild-type hearts at 12 months of age (Figure 3C) when treated with and without bafilomycin A1 treatment, using doses previously described to accumulate stop lysosomal degradation $^{23,42,43}$ (Figure 3D). Quantitative measurement of genes involved in the autophagic process (mRNA) was then analyzed for evidence of altered autophagy (Figure 3E). No differences in Atg5, Atg7, Atg12, Bnip3, LC3, or Vps34 mRNA were identified compared to wild-type hearts (Figure 3E). Although mutations in other myofibrillar proteins causing cardiomyopathy enhance autophagy, the Bag3 P209L mutation did not alter this lysosomal degradation 
A

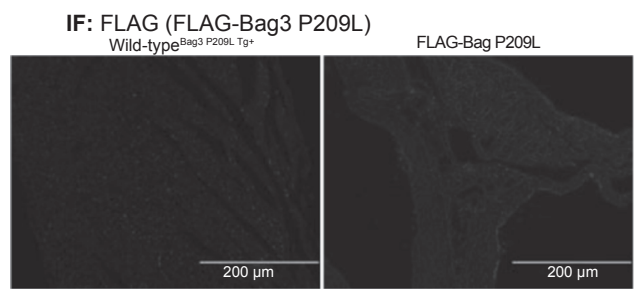

C
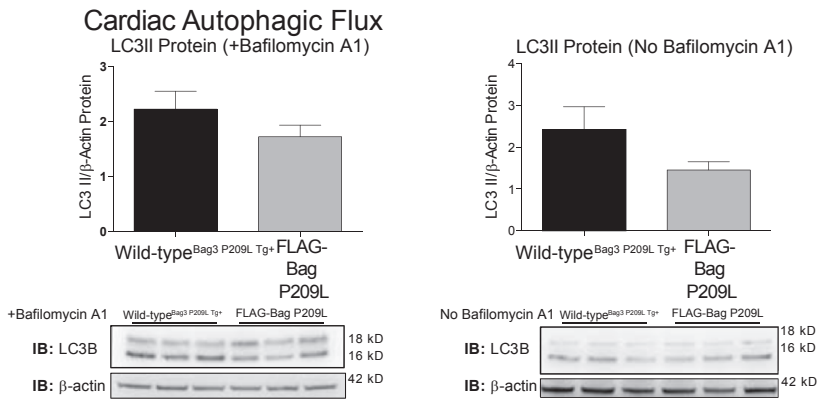

E

Autophagy Gene Expression
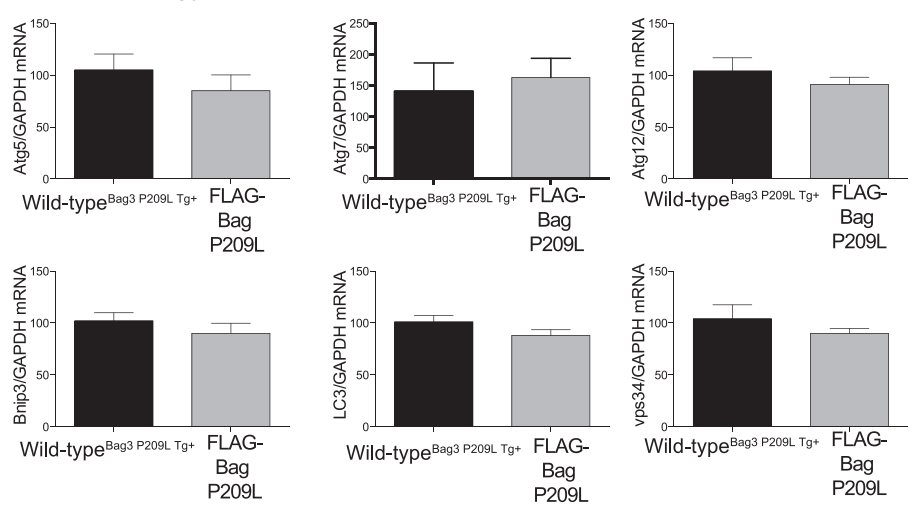

B

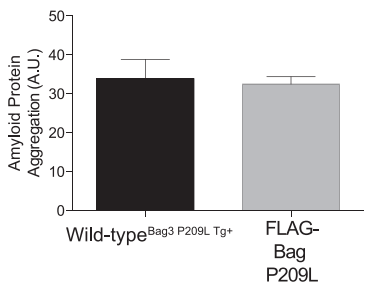

D

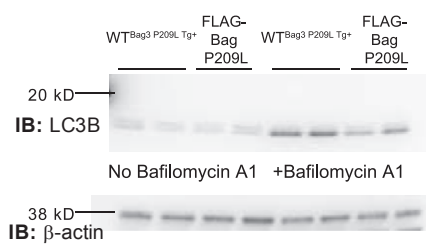

Figure 3 Analysis of protein aggregate and autophagic flux in $\alpha$ MHC-Bag3 P209L transgenic hearts at 12 months. A: Immunofluorescence detection of FLAG-Bag3 P209L in transgenic, but not wild-type, hearts. B: Colorimetric staining of protein aggregation. C: Western blot analysis of cardiac autophagic flux from $\alpha$ MHC-Bag3 P209L and littermate wild-type mice at 12 months of age. D: Western blot analysis demonstrating that bafilomycin induction is adequate. E: Real-time quantitative RT-PCR analysis of Atg5, Atg7, Atg12, Bnip3, LC3, and Vps34, mediating autophagy. A t-test was used to compare genotypes at each time point. Measurements represent means \pm SEM (B, C, and $\mathbf{E}) . N=6$ per group (B and $\mathbf{E}) ; N=3$ per group (C). IB, immunoblot; IF, immunofluorescence. pathway significantly. Alterations in cardiac function do not appear to be related to changes in autophagic flux in this model.

An essential underlying mechanism of heart failure is the loss of myocytes by apoptosis. ${ }^{44,45}$ In tumor cell lines, silencing of Bag3 enhances apoptosis, whereas increasing Bag3 inhibits spontaneous or drug-induced apoptosis. ${ }^{37,46-53}$ Different mechanisms have been identified in cancer, including Bag3's protection of IKK $\gamma$ from proteasome delivery promoting cell survival. ${ }^{37}$ We next investigated the potential role of apoptosis in the Bag3 P209L $\mathrm{Tg}^{+}$hearts by measuring cardiac apoptosis by TUNEL and AIF immunofluorescence (Figure 4A). TUNEL positivity was investigated in both fibroblasts (TUNEL+, vimentin+) and in nonfibroblast cells (TUNEL+, vimentin negative) made up primarily of cardiomyocytes. Significantly less apoptosis (TUNEL+) was seen in the Bag3 P209L $\mathrm{Tg}^{+}$fibroblasts (Figure 4A), whereas Bag3 P209L Tg ${ }^{+}$cardiomyocytes (TUNEL+ and vimentin negative cells) trended higher compared to sibling wild-type controls at 1 year of age (Figure 4B). No differences in AIF staining were seen in between Bag3 P209 $\mathrm{Tg}^{+}$hearts and sibling-matched wild-type controls (Supplemental Figure S2). This led us to further analyze the fibroblast (Vimentin+) characteristics histologically (Figure 4B). Unexpectedly, we identified that the Bag3 P209L $\mathrm{Tg}^{+}$hearts exhibited more vimentin+ cells compared to wild-type hearts (Figure 4B). Hearts were analyzed in quadrants as outlined in Figure 4C, with quadrant 2 and quadrant 3 (Q2 and Q3) including primarily the left ventricle and quadrant 1 and quadrant 4 (Q1 and Q4) making up the right and upper left ventricle and septum. Blinded quantitative analysis (C.C.Y.) of vimentin (+) cells from multiple animals identified a consistent and significant increase in the left ventricle (Figure 4A), which was distinctly different from that found in Q1 and Q4 (Supplemental Figure S3). Identification of vimentin in parallel studies of cardiac ventricles in Bag3 P209L Tg+ hearts at 12 months illustrates that approximately fivefold more vimentin is present (Figure 4D). Qualitatively, these vimentin (+) cells in the Bag3 P209 $\mathrm{L} \mathrm{Tg}^{+}$ hearts were distributed around the vasculature, which may represent smooth muscle or fibroblast pericytes (Figure 4B). This vascular distribution of vimentin $(+)$ cells was not seen in any of the age-matched wild-type controls (Figure 4B). Together, these findings indicate that the Bag3 P209L $\mathrm{Tg}^{+}$ hearts do not have increases in cardiomyocyte apoptosis at 12 months of age, but have an enhanced fibroblast presence in the 
A IF: TUNEL and Vimentin Co-Stained

TUNEL Positive Non-Fibroblast Cells (Vimentin Negative, Mainly

Cardiomyocytes)

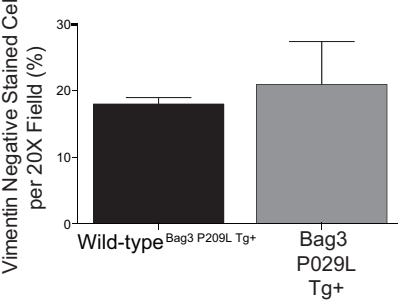

TUNEL Positive Fibroblasts

(Vimentin Positive)

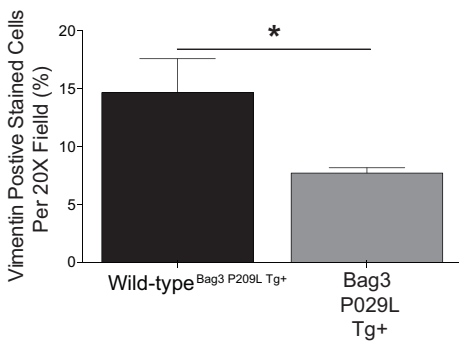

B Vimentin Staining Cardiac Sections IHC

\section{Quadrant 2 (Q2)}
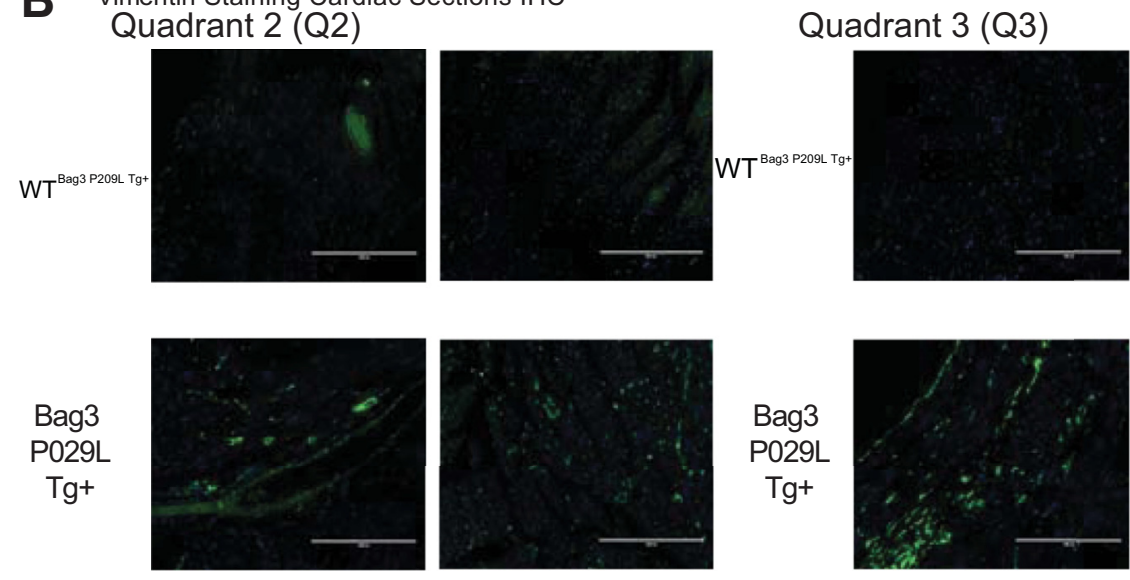
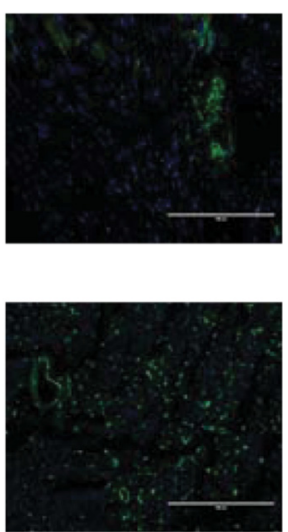

C Vimentin Staining IHC Quantification

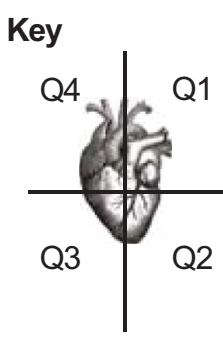

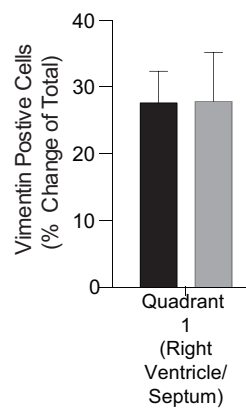

Wild-type Bag3 P209L Tg+ Bag3 P029L Tg+

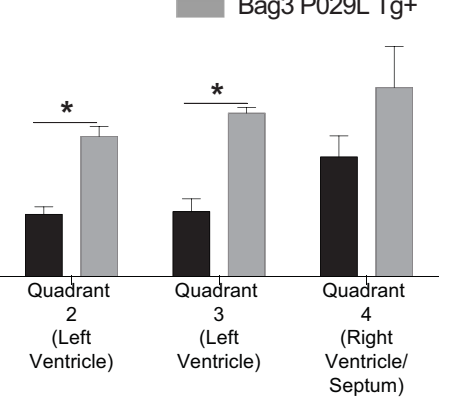

D

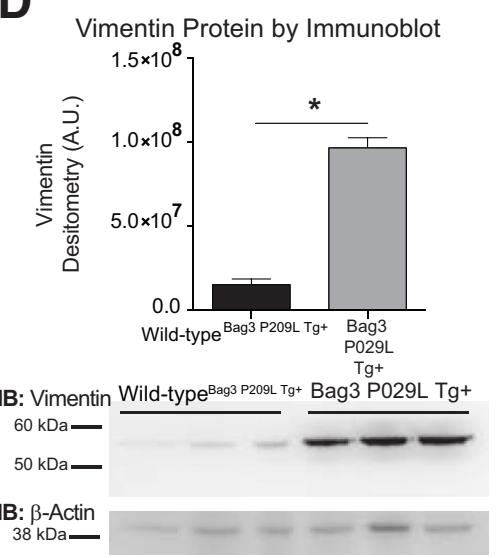

Figure 4 aMHC-Bag3 P209L transgenic hearts have increased left ventrical (LV) fibroblast areas in a perivenular distribution and decreased fibroblast apoptosis. A: Costaining of TUNEL positive nonfibroblast (left) and fibroblast (vimentin positive) cells by immunohistochemistry. B: Representative confocal immunofluorescence images of cardiac fibroblasts from histological sections of $\alpha$ MHC-Bag3 P209L and littermate wild-type mice at 12 months of age. Shown are quadrant 2 and quadrant 3, tracking with the LV (quadrant 1 and quadrant 1 primarily right ventricle). C: Nonbias quantitative logarithmic analysis of vimentin $(+)$ cell staining in quadrants 1 to 4. D: Western immunoblot analysis of cardiac vimentin in Bag3 P209L hearts compared to wild-type hearts at 12 months of age. A t-test was used to compare genotypes at each time point. Measurements represent means \pm SEM $(\mathbf{A}) . N=12\left(\mathbf{A}\right.$, wild-type $^{\mathrm{Bag} 3} \mathrm{P} 209 \mathrm{~L} \mathrm{Tg}+\mathrm{f}$; $N=6$ (A, Bag3 P209L Tg ${ }^{+}$cardiac sections). ${ }^{*} P<0.05$ versus age-matched sibling wild-type mice. Scale bars $=200 \mu \mathrm{m}(\mathbf{B})$. IHC, immunohistochemistry; TUNEL, terminal deoxynucleotidyl transferase-mediated dUTP nick-end labelling. AU, arbitrary units; IB, immunoblot; IF, immunofluorescence.

left ventricle and an increase in pericytes. With the ability to inhibit vessel formation and induce vessel dissociation, ${ }^{54}$ their role in the heart has been attributed to their contributions to tissue repair. $^{55,56}$

Recent studies have linked the presence of pre-amyloid oligomers to cardiac dysfunction and heart failure.
Mutations in the cardiac myofibrillar protein CryAB, another small heat shock protein, have been shown to result in the accumulation of proteotoxic misfolded protein, characterized by reactivity to an amyloid recognizing antibody. ${ }^{17,18,57}$ Interestingly, antibodies recognizing amyloid conformations have been shown to react similarly in 
multiple causes of human heart failure. ${ }^{17}$ Subsequently, cardiac expression of a misfold prone protein [polyglutamate 83 (PQ83) repeat] was compared to the cardiac expression of a protein not prone to misfold (PQ19 repeat) to demonstrate the proteotoxicity of these proteins in cardiomyocytes. ${ }^{16}$ The resulting PQ83 mice progressed rapidly into heart failure and death, whereas the PQ19 were entirely unaffected. ${ }^{16}$ The PQ83 hearts had cardiomyocyte accumulation of the preamyloid oligomers (PAOs), whereas the PQ19 did not, illustrating the causative role of the misfolded proteins in heart failure that are identified as PAOs. ${ }^{16}$ This led us to hypothesize that the Bag3 P209 $\mathrm{Lg}^{+}$heart failure may be mediated by the accumulation of PAO. Immunohistochemistry of PAO (recognized by the A11 antibody) colocalizing with cardiomyocytes (recognized by the MF20 antibody) was performed on Bag3 P209 $\mathrm{Lg}^{+}$heart sections (Figure 5A). Although gross histological analysis did not reveal overt differences in the Bag3 P029L Tg + hearts (Figure 5B), differences in infiltrating immune cells were detected on higher-power evaluation (Figure 5C). When quantified by nuclei counts using an algorithm recognizing nuclei as a function of area, significantly more nuclei were present in the Bag3 P029L Tg + hearts (Figure 5D), consistent with an increase in infiltrates, likely immune cells. Several hypotheses have emerged concerning the cellular mechanisms by which misfolded proteins trigger inflammation, including the direct activation of the inflammasome by misfolded proteins (eg, amyloid- $\beta$, amylin, serum amyloid A, and prions) that could trigger downstream IL-1, caspase activation, and heart failure. ${ }^{58}$

In neurodegenerative diseases where amyloidogenic proteins, including PAOs, mediate proteotoxicity, abnormal mitochondrial dynamics composed of fission and fusion events have been reported. ${ }^{59,60}$ One of the distinctive findings by TEM of the Bag3 P209 $\mathrm{Tg}^{+}$hearts was the apparent dysregulation of the mitochondrial sizes, briefly illustrated in Figure 2D. We next analyzed the TEM images in more detail, assaying sections throughout the ventricles of the Bag3 P209L $\mathrm{Tg}^{+}$and wild-type hearts (Figure 6). We identified an apparent increase in mitochondria by manual counting of TEM images (Figure 6A), but only mild decreases in area (Figure 6B). These manual counts were complemented by quantitative analysis of mitochondrial genes normalized to a nuclear intron (H19) (Figure 6C). Although mitochondrial NADH dehydrogenase 1 (ND1), cytochrome-b (Cytb), and cytochrome-c oxidase 1 (CO1) were increased in the Bag3 P209L hearts, significant increases were found only in ND1, consistent with a nearly doubling of the mitochondrial number (Figure 6C). Mitochondrial dynamics are regulated by a number of key proteins encoded by mitofusins (Mfn1, Mfn2) and optic atrophy (Opa1) to regulate mitochondrial fusion and dystrophin-related protein 1 (Drp1) and fission 1 homolog (Fis1) that regulate mitochondrial fission. ${ }^{61}$ We identified that the Bag3 P209 $\mathrm{Lg}^{+}$hearts had significant decreases in the mitochondrial fusion Opa1 mRNA (Figure 6D) and the mitochondrial fission Drp1 mRNA (Figure 6E). No differences in Bag3 P209L cardiac Mfn1 or Fis1 were present compared to wild-type hearts (Figure 6, D and E). With an increased number of smaller mitochondria, Bag3 P209L $\mathrm{Tg}^{+}$hearts exhibited significant alterations in Opal and Drp1 involved in regulating fusion and fission, respectively.

Alterations in mitochondria led to a nontargeted metabolomics analysis to determine any gross metabolic changes in the Bag3 P029L $\mathrm{Tg}+$ hearts at 12 months of age (Figure 7A). By principal components analysis, the Bag3 P029L Tg + hearts had a distinct signature from the wildtype hearts (Figure 7B), with 11 metabolites significantly different by $t$-test (Figure $7 \mathrm{C}$ and Table 3). A partial least squares discriminant analysis was also run to differentiate the two groups (Figure 7D) by identifying unique metabolites of interest, on the basis of their known relationships indicated by their VIP score (Figure 7E). Together, these studies found that Bag3 P209L $\mathrm{Tg}^{+}$hearts had elevated aspartic acid and dehydroascorbic acid and decreased taurine, O-methylphosphate, and adenosine-5monophosphate (AMP). Pathway enrichment analysis of both $t$-test and VIP identified metabolites found pathways involved in D-glutamine metabolism, taurine metabolism, and alanine, aspartate, and glutamate metabolism had the highest impact (Supplemental Figure S4A and Supplemental Table S4). Disease association enrichments identified that these metabolites were most closely associated (lowest $P$ value) with the heart failure metabolomics reference set, continuous ambulatory peritoneal dialysis, and tumor-bearing mice (Supplemental Figure S4B). These metabolites also tracked with locations within the cell involved in mitochondria, peroxisome, and skeletal muscle (lowest $P$ value) (Supplemental Figure S4C).

Recent studies have identified that Bag3 mutations in dilated cardiomyopathy are predicted to result from loss-offunction mutations, in which knock-down of Bag3 in a zebrafish model recapitulated the observed heart failure. ${ }^{8}$ Because these studies implied that the loss of function, also known as haploinsufficiency, was because of the Bag3 mutations directly, we hypothesized that the Bag3 P209L transgene would have no effect on the endogenous wildtype Bag3 levels. Because the Bag3 P029L Tg+ mouse model described herein used a human Bag3 P209L transgene, we next assayed the endogenous levels of (mouse) Bag3 at 1 year of age. To our surprise, the endogenous Bag3 P209L mRNA levels (measured by qPCR of mouse Bag3 mRNA spanning exons 2 to 3 ) were suppressed to $<20 \%$ of wild-type heart levels (Figure 8A) with evidence of the human Bag3 P209L present (Figure 8B). This paralleled decreased levels of total Bag3 protein recognizing both mouse and human P209L Bag3 (Figure 8C). There is limited information on how Bag3 is regulated transcriptionally, but involvement of the transcription factors cJun/ cFos (AP1), NF- $\kappa$ B, and HSF1 has been reported and may be related to the observed findings. ${ }^{50,62-65}$ 
A

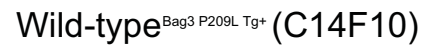

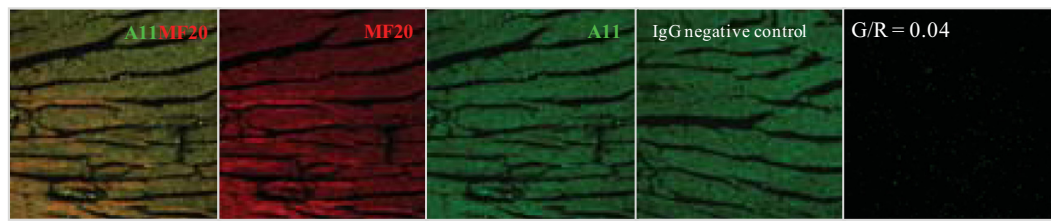

Bag3 P209L Tg+ (C11F01)
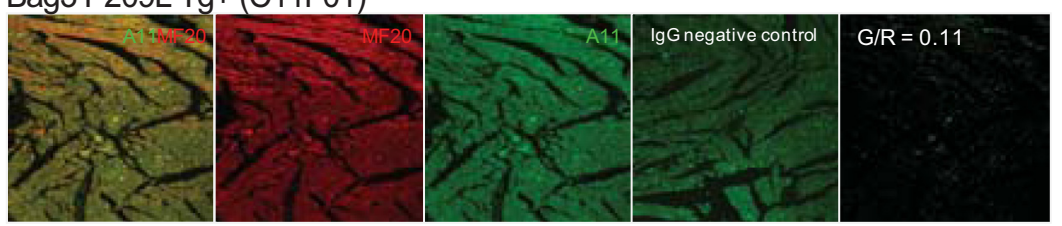

B

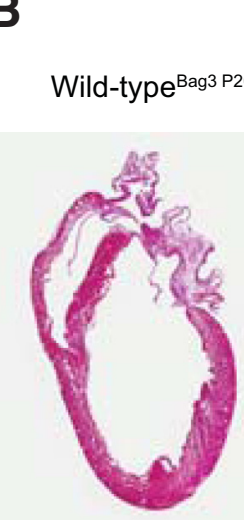

C11 F01
C Nuclei by Algorithm Count

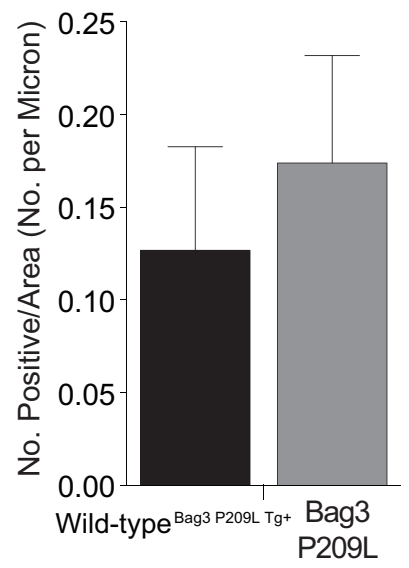

$\widehat{\widetilde{x}}$ PAO Positive Cardiomyocytes

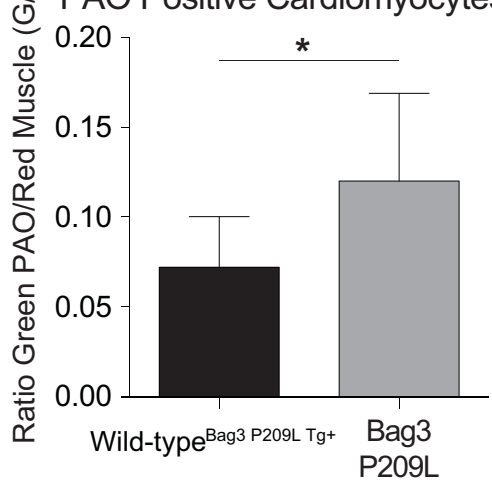

$\mathrm{Tg}+$
D Representative H\&E

Wild-type Bag3 P209L Tg+ $^{-}$

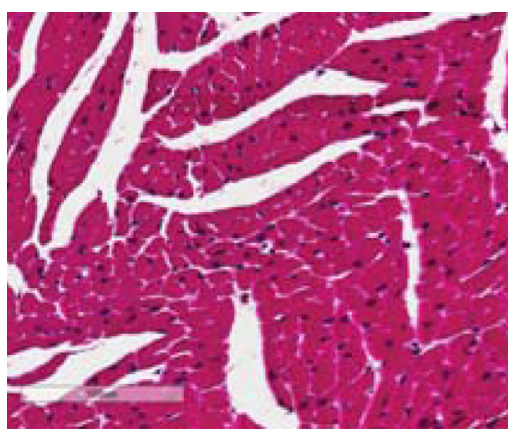

E Representative H\&E Bag3 P209L Tg+

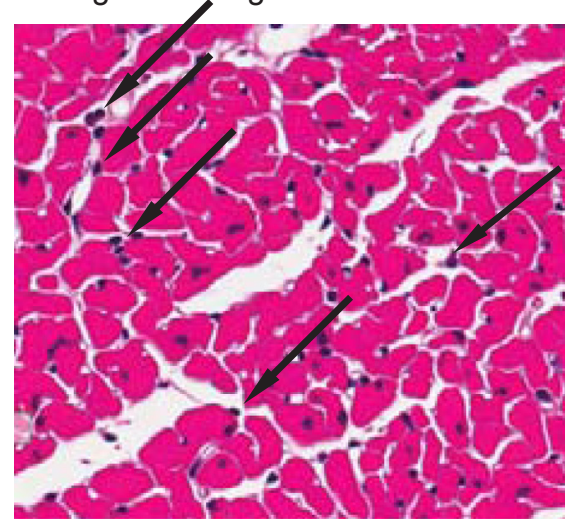

\section{Bag3 P209L Tg+}

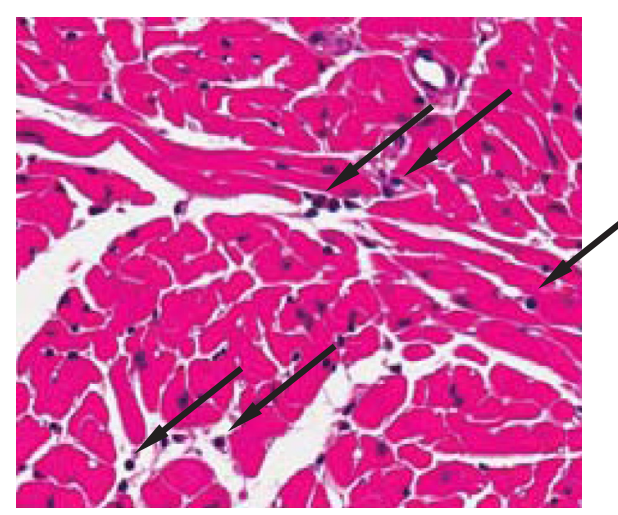

Bag3 P209L Tg+

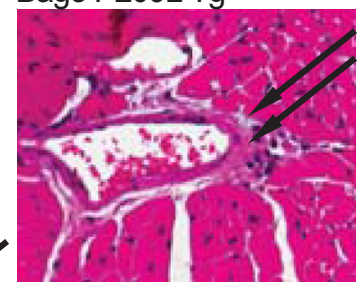

Bag3 P209L Tg+

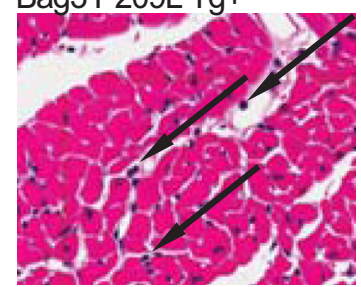

Figure 5 Analysis nuclei in $\alpha$ MHC-Bag3 P209L transgenic hearts at 12 months. A: Immunohistochemistry analysis of preamyloid oligomers (PA0; anti-A11 antibody), cardiomyocytes (anti-MF20 antibody), and IgG controls showing representative green (PAO) as a percentage of red (cardiomyocytes) in cardiac sections. B: Gross comparison of $\alpha$ MHC-Bag3 P209L and littermate wild-type mice [hematoxylin and eosin (H\&E) stained). C: Algorithmic analysis of nuclei quantitated per area of cross-sectional area. D: Representative Bag3 P209L $\mathrm{Tg}^{+}$hearts and age-matched sibling wild-type hearts with cellular infiltrates.

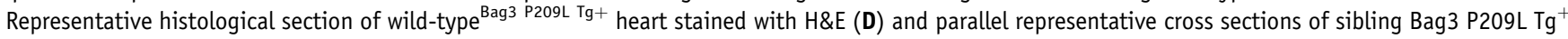
hearts with inflammatory cell infiltrates (arrows) at 12 months of age (E). A t-test was used to compare genotypes at each time point. $N=3$ Bag3 P209L Tg ${ }^{+}(\mathbf{A})$; $N=4$ Bag3 P209L Tg ${ }^{+}$biological replicates, 3 cross-sectional areas each $(\mathbf{A}$ and $\mathbf{B}) ; N=5$ wild-type ${ }^{\mathrm{Bag} 3 \mathrm{P} 209 \mathrm{~L} \mathrm{Tg}+}(\mathbf{A}) ; N=3$ wild-type ${ }^{\mathrm{Bag} 3 \mathrm{P} 209 \mathrm{~L} \mathrm{Tg}+}(\mathbf{C}) .{ }^{*} P<0.05$ versus age-matched sibling wild-type mice. Original magnification: $\times 20(\mathbf{A}, \mathbf{D}$, and $\mathbf{E}) ; \times 0.7(\mathbf{B})$. 


\section{A}

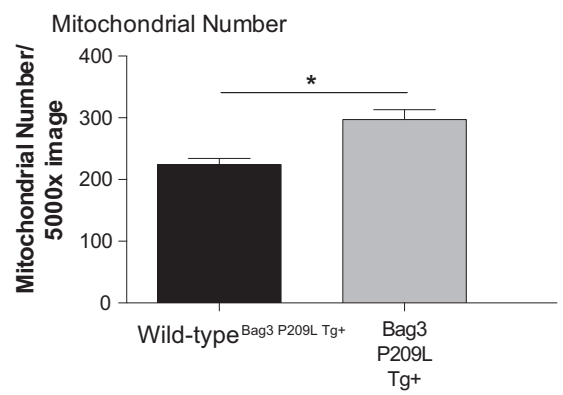

B

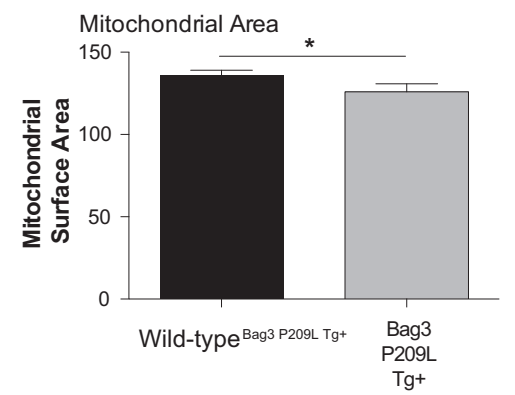

C

qPCR mt-DNA Normalized to nu-DNA
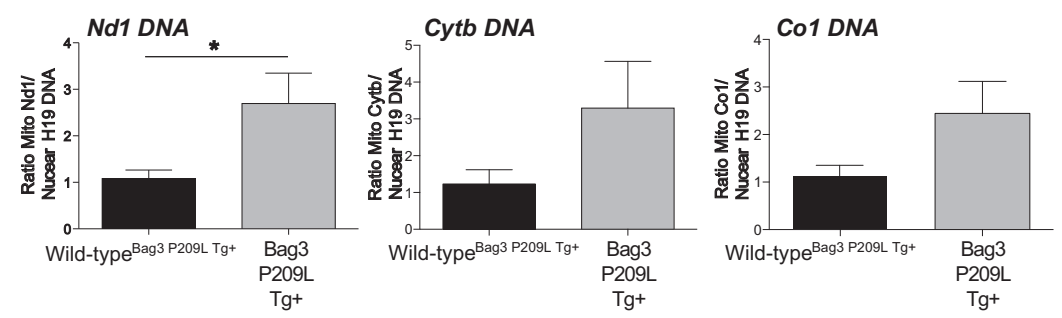

D RT-qPCR Mitochondrial Fusion Genes

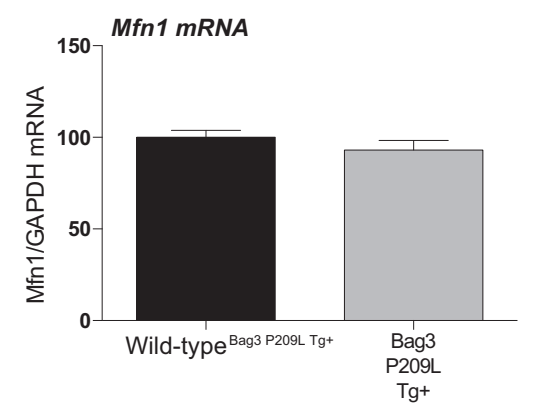

$\mathbf{E}$

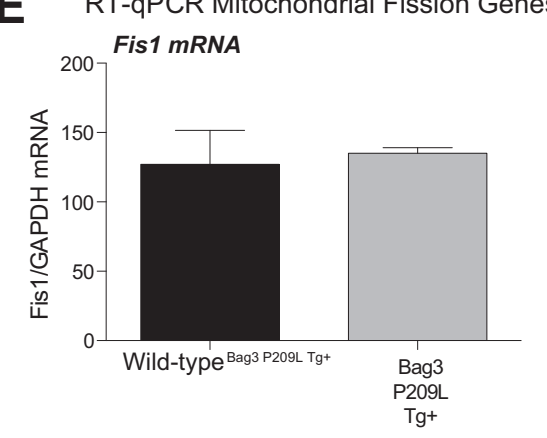

The Bag3 P209L transgenic hearts had several notable parallels with neurodegenerative disease, including an increase in pre-amyloid oligomers and a dysregulation of mitochondrial dynamics. ${ }^{59,60}$ We hypothesized further that the Bag3 P209L $\mathrm{Tg}^{+}$hearts exhibited p38 activation and an increase in ubiquitinated proteins, on the basis of recent discoveries in models of neurodegenerative diseases. Misfolded protein aggregates have been reported in aged Drosophila to activate p38 signaling ${ }^{66}$ and in neurons to activate both the unfolded protein response and p38

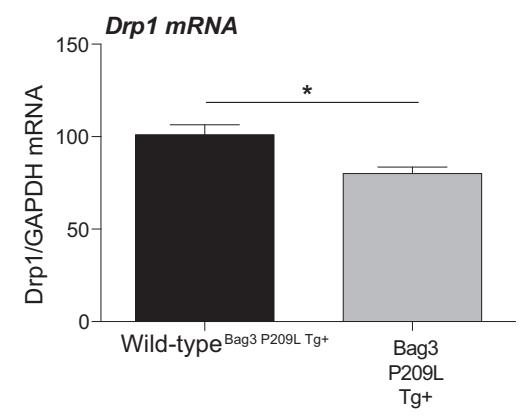

Figure 6 aMHC-Bag3 P209L transgenic hearts exhibit alterations in mitochondria number and dynamics. Quantitative analysis of mitochondrial number (A) and mitochondrial area (B) in $\times 5000$ TEM sections. C: Quantitative analysis of the mean cytochrome c oxidase subunit 1 ( $\mathrm{CO} 1$, aka mtC01)/cytochrome b (Cyt-b, aka mt-Cyb)/NADH dehydrogenase 1 (ND1, aka mt-nd1) normalized to nuclear $\mathrm{H} 19$ (imprinted maternally expressed transcript, non-protein coding) of aMHC-Bag3 P209L and littermate wild-type mice at 12 months of age. Real-time quantitative RT-PCR (RT-qPCR) analysis of genes regulating mitochondrial fusion (mitofusin 1/Mfn1 and optic atrophy1/opa1) (D) and mitochondrial fission (mitochondrial fission 1 protein/Fis1 and dynamic-related protein 1/Drp1) (E). A $t$-test was used to compare genotypes at each time point. Data are expressed as means \pm SEM. $N=4\left(\right.$ A and B, Bag3 P209L Tg $\left.{ }^{+}\right) ; N=5$ per group (C); $N=6$ per group (A and $\mathbf{B}$, wildtype ${ }^{\text {Bag3 } \mathrm{P} 209 \mathrm{~L} \mathrm{Tg}+}$, and $\mathbf{D}$ and $\left.\mathbf{E}\right)$. ${ }^{*} P<0.05$ versus age-matched sibling wild-type mice. nu-DNA, nuclear DNA; qPCR, real-time quantitative PCR.

signaling. ${ }^{67,68}$ Analysis of phospho-p38 protein identified that Bag3 P209 $\mathrm{Tg}^{+}$hearts exhibited significant increases in activated p38 compared to wild-type hearts at 1 year (Figure 8D), with modest increases in ubiquitinated proteins (Figure 8E). Targeted activation of p38 mitogen-activated protein kinase in cardiomyocytes has been shown to induce heart failure, having negative inotropic and restrictive diastolic effects. ${ }^{69}$ Activation of p38 in cardiomyocytes has also been implicated in the inflammatory response, including IL-1b-induced COX-2 expression, PGE2 
A

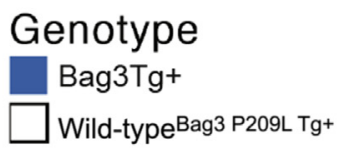

Genotype

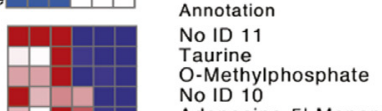

No ID 10

Adenosine-5'-Monophosphate

Adenosine 2

Aspartic Acid

Linoleic Acid

Oleic Acid

Glycine

O-Phosphocolamine

O-Phosphocolamine

2-Hydroxybutyr
No ID 17

Fumaric Acid

Fumaric

No ID 8 acidamic Acid 1

Urea 1
Beta-Hydroxybutyric Acid

Alanine

No ID 2

No ID 16
No ID 4

Palmitic Acid

Malic Acid

No ID 19

Maltose

Stearic Acid

Threonine 2

Succinic Acid
Adenosine 1

Pdenosine 1

Lysine 1

Campesterol

Beta-Monostearin

Cholesterol

ene-1-2-4-Triol/Hypotaurine

Myristic Acid Deuterated Std

Myoinositol

No ID 13

Benzoic Acid

Alpha-Monopalmitin

Phosphoric Acid

Creatinine

No ID 15

Alpha-Monostearin

No ID 20
No ID 7

No ID 7
No ID 5

Disaccharide 2

No ID 23

2-Hydroxypyridine

No ID 9

Glycerol 1-Phosphate

Threonine 1

Gluconic Acid Or Similar Sugar Acid

Ethylglycine

Disaccharide 1

Valine 1

Alpha-Tocopherol

Serine 1

Fructose-6-Phosphate

Glucose And Other Aldohexoses

Glucose And Other Aldohexoses

Citric Acid/Isocitric Acid

Serine 2

roxyglutaric Acid

No ID 3

Dehydroascorbic Acid

Pimilar 1

Pantothenic Acid

Glucose-6-Phosphate Or Similar 3

Glutamic Acid 3

Glucose-6-Phosphate Or Similar 2

Aspartic Acid

No ID 12

Ribulose-5-Phosphate/Xylulose-5-Phosphate

Lysine 2

No ID 1

C13 Hydrocarbon

No ID 21

\section{B Principal Components Analysis}

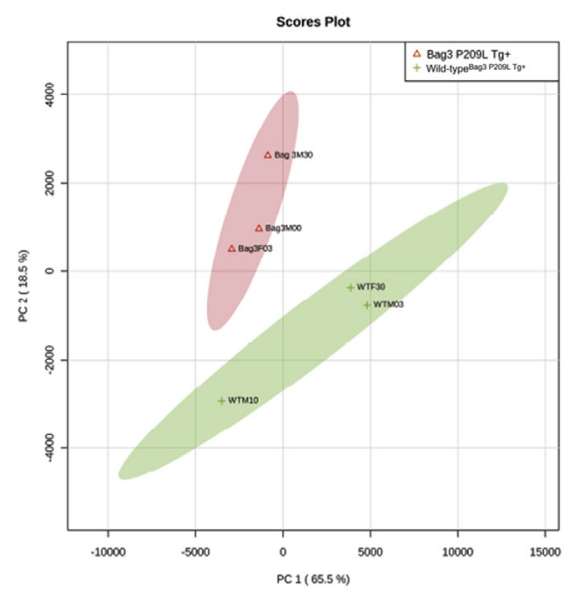

Fold Change (vs. WT

$10935.0664 * *$

17.1038

10.121
1.9967

1.7998

1.6779
1.6693

1.6693
1.6533

1.6533
1.3736

1.3644

1.3292

1.3268

1.2187

1.179

1.179

1.0025

.9952

0.9859

0.9822

0.982

0.969

0.9683
0.9656

0.9656

0.9548
0.9523

0.9523
0.9385

0.9385
0.9384

0.9384
0.9101

0.9101
0.9079

0.9079
0.8567

0.8567
0.8405

0.8316

0.8235

0.812

0.8129

0.806

0.7962

0.7962
0.7922

0.7892

0.789
0.7809

0.7809

0.7717
0.7676

0.7676
0.7613

0.7613
0.7564

0.7564
0.7556

0.7556
0.754

0.754
0.751

0.751
0.7503

0.7502

0.7458

0.7347
0.7286

0.7286

0.7227

0.7032
0.6985

0.6985
0.6928

0.6498

0.6211

0.6103

0.5951

0.5847

0.5836

0.5689

0.5547

0.5153

0.5082

0.4921

0.4914

0.4711

0.4559

0.4393

0.4393
0.4377

0.4366

0.4151

0.405

0.3549
0.3539

0.3539
0.2944

0.2944
0.2728

0.2728
0.2713

0.2713
$0.000091 * *$
$0.000067 * *$

$0.000067 * *$

C

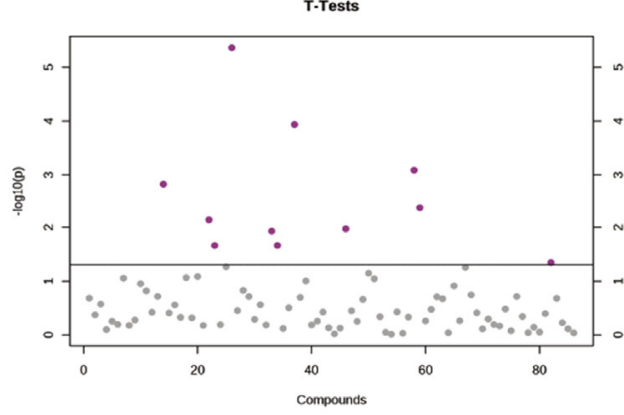

D PLS-DA Analysis

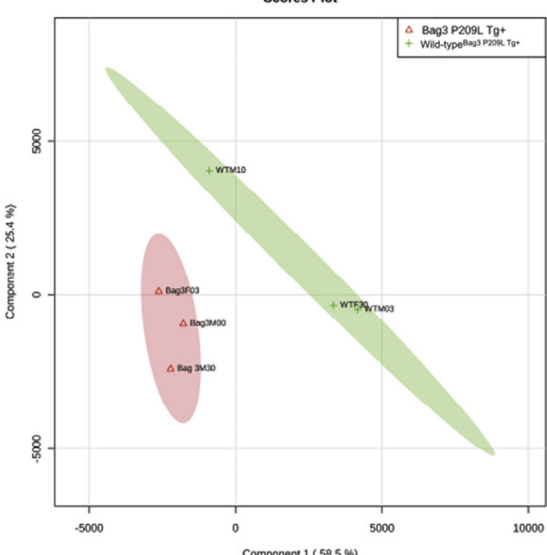

E PLS-DA VIP Analysis

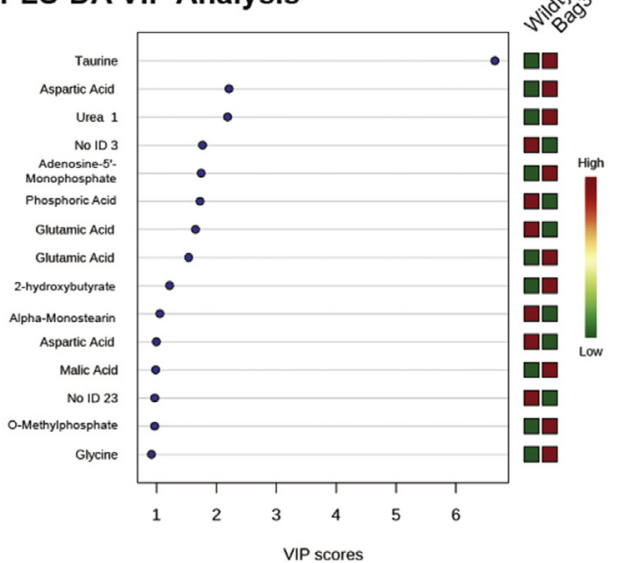


Table 3 t-Test Significant Metabolites Identified by Nontargeted Metabolomics Analysis of Bag3 P029L Tg + Heart versus SiblingMatched Wild-Type ${ }^{\text {Bag3 P209L Tg Controls }}$

\begin{tabular}{lll}
\hline$t$-Test significant & $P$-value & FDR \\
\hline No ID 11 & $4.33 \times 10^{-6}$ & 0.00037239 \\
Aspartic acid 1 & 0.0001159 & 0.0049839 \\
No ID 21 & 0.00081798 & 0.023449 \\
0-Methylphosphate & 0.0014924 & 0.032087 \\
Dehydroascorbic acid & 0.0043074 & 0.074088 \\
C13 Hydrocarbon & 0.0072195 & 0.10348 \\
Taurine & 0.010598 & 0.12587 \\
Aspartic acid 2 & 0.011709 & 0.12587 \\
No ID 14 & 0.021674 & 0.18684 \\
No ID 10 & 0.021726 & 0.18684 \\
Adenosine-5'-monophosphate & 0.045195 & 0.35334 \\
\hline
\end{tabular}

Identification of metabolites indicated in Figure $7 C . N=3$ hearts per group. FDR, false discovery rate.

biosynthesis. $^{70}$ Together, these findings suggest that the Bag3 P209L drives an increase in cardiomyocyte PAOs, which leads to p38 activation and increase protein ubiquitination, as is seen in neurodegenerative diseases in vivo.

\section{Discussion}

The 575 amino acid BAG3 protein is expressed predominantly in the heart and skeletal muscle and functions as a cochaperone with members of the heat shock protein family to facilitate multiple important cellular processes, including the removal of misfolded and degraded proteins, apoptosis by interacting with $\mathrm{Bcl} 2$, the structural integrity of the Zdisk by binding CapZ, and chaperone-associated selective autophagy. ${ }^{71}$ Single allele mutations in BAG3 have been associated with adult onset hereditary dilated cardiomyopathy. Significant decreases in BAG3 have been identified in end-stage failing human heart and in animal models of heart failure secondary to myocardial infarction and pressure overload. ${ }^{71}$ Herein, we report the first mammalian model of a single amino acid mutation of BAG3 (P209L) in exon 3 of Bag3 associated with the development of muscle disease with left ventricular dysfunction and heart failure first reported in $2009 .{ }^{10}$ We identified previously unreported underlying mechanisms, including altered mitochondrial dynamics (increased fragmented mitochondria), the presence of pre-amyloid oligomers, the development of wildtype BAG3 haploinsufficiency, and activation of p38 signaling, paralleling the emerging pathogenesis in other diseases of misfolded proteins, such as Alzheimer and Huntington diseases.

Many of the findings in the present study may be related to domain P209L mutation, as the domain is linked to the cell's general ability to clear misfolded proteins. BAG3 has five domains: i) the WW domain (with two tryptophan motifs = WW) (amino acids 21 to 55); ii) the IPV motif 1 (amino acids 87 to 101); iii) the IPV motif 2 (amino acids 200 to 213, where P209L is located); iv) the PXXP motif (amino acids 302 to 412); and v) the BAG motif (amino acids 420 to 499). Each of these domains binds specific proteins, each with distinct cellular processes. In the Nterminal end, the WW domain is involved in the induction of autophagy, IPV motifs (1 and 2) bind HSPB8 (and to a lesser extent, HSPB6), the Bcl2 binds the region around amino acid 440, and HSC70, HSP70, and CapZ bind the cterminal BAG domain. ${ }^{12,71,72}$ Because the P209L mutation affects the IPV motif (amino acids 200 to 213), our findings that the Bag3 P209L hearts did not have altered autophagy (Figure 3) or apoptosis (Figure 4) mediated by the WW (amino acids 21 to 55) and $\mathrm{Bcl} 2$ binding region (amino acids 440), respectively, were not surprising because they are not related to this region. Similarly, a recent report identified that Bag3 P209L was as effective as wild-type BAG3 in enhancing LC3 lipidation and maintaining autophagic flux. ${ }^{73}$ Similarly, the lack of any alterations in the Bag3 P209 $\mathrm{Lg}^{+}$heart sarcomeres (Figure 2D) is also consistent with the recent finding that the Bag3 P209L is able to rescue the myofibrillar disintegration found when Bag3 is knocked down in zebrafish models, ${ }^{13}$ demonstrating that the mutation does not have dominant negative activity and maintains structural integrity. More important, the IPV domains (where P209L is located) associates with critical HSPB proteins found necessary to degrade the Huntington disease-causing Htt43Q mutation, caused by its misfolding (ie, PAO) and proteotoxicity. ${ }^{72}$ Taken together, these findings implicate the Bag3 P209L mutation in producing a protein more prone to forming PAOs, or by altering the cells' ability to clear PAOs, or possibly both. Either way, the altered physiology leading to heart failure identified in the current study is mechanistically consistent with a proteotoxic mechanism in vivo.

Although the formation of pre-amyloid oligomers (Figure 5A) in the $\alpha$ MHC-Bag3 P209L parallel those seen in other small heat shock protein mutations, notably the CryAB R120G, many differences exist in the cardiac phenotypes that these mutant and native proteins have in vivo. At 12 months, the Bag3 P029L Tg + heart does not have any

\footnotetext{
Figure 7 Nontargeted metabolomics analysis of $\alpha$ MHC-Bag3 P209L transgenic hearts at 12 months of age. A: Heat map representation of metabolites identified in $\alpha$ MHC-Bag3 P209L and littermate wild-type hearts at 12 months of age. B: Principal components (PC) analysis of $\alpha$ MHC-Bag3 P209L and littermate wild-type hearts. C: $t$-Test analysis of metabolites, including significant metabolites (above horizontal line), listed below with $P$-value and false discovery rate (FDR) estimate. D: Partial least squares discriminant analysis (PLS-DA) score plot demonstrating significant separation between groups. E: The variable influence on the projection (VIP) parameter to select variables that have the most significant contribution in discriminating between metabolomics profiles in a PLS-DA model. Statistical analyses were performed as described in Materials and Methods. Double asterisks denote fold-change inaccurate because of imputing a non-zero value resulting from the metabolite not being detected in one group. $N=3$ biological replicates per group (A-E). WT, wild-type.
} 
A

Mouse Bag3 mRNA in Heart at 12 Months

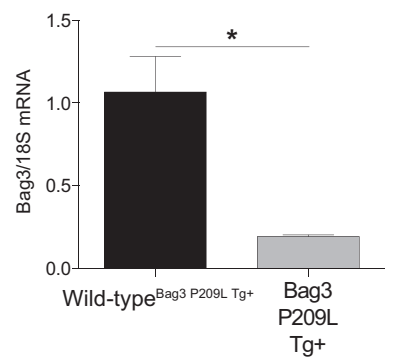

C Total Mouse and Human Bag3 Protein in Heart at 12 Months
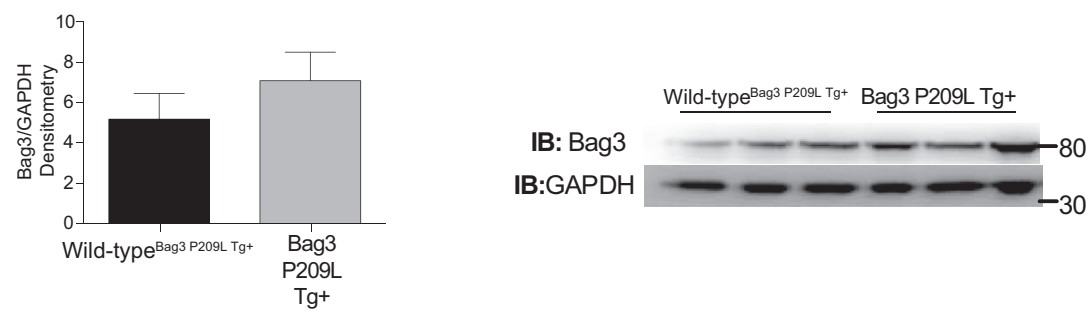

D Phospho p38/Total p38 Protein in Heart at 12 Months

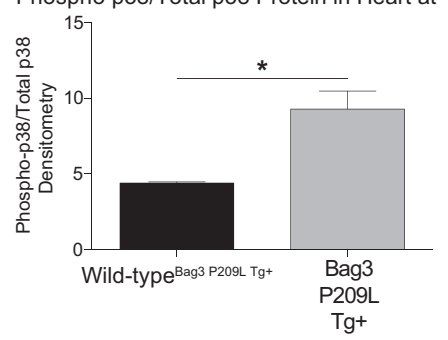

\section{IB: $P$}

B: Total p38

IB: $\beta$-Actin

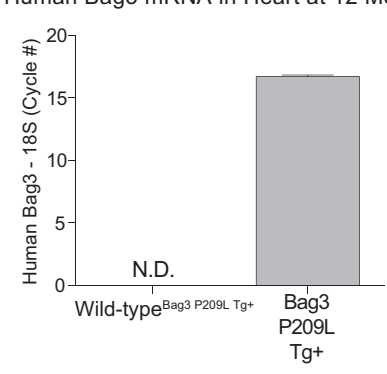

g+

Figure 8 aMHC-Bag3 P209L down-regulates wild-type BAG3 and activates P38 at 12 months of age. Cardiac expression of endogenous mouse Bag3 (A) and human Bag3 (B) mRNA expression analysis by real-time quantitative RT-PCR. Western blot analysis of total (mouse and human) Bag3 protein (C), phospho-p38/p38 (D), and ubiquitinated proteins from $\alpha$ MHC-BAG3 P209L and littermate wild-type mice (E) at 12 months of age. A $t$-test was used to compare genotypes at each time point. Measurements represent means \pm SEM $(\mathbf{A}-\mathbf{E}) \cdot N=4\left(\mathbf{A}\right.$, wild-type $\left.{ }^{\mathrm{Bag} 3 \mathrm{P} 209 \mathrm{~L} \mathrm{Tg}+}\right) ; N=5(\mathbf{A}$ and $\mathbf{B}$, Bag3 P209L Tg$) ; N=3$ per group (C and D); $N=6$ per group (B, wild-type ${ }^{\text {Bag } 3 \text { P200L } \mathrm{Tg}+}$, and E). ${ }^{*} P<0.05$ versus age-matched sibling wild-type mice. IB, immunoblot; ND, not detected.

Ubiquitinated Protein Concentration in Heart at 12 Months

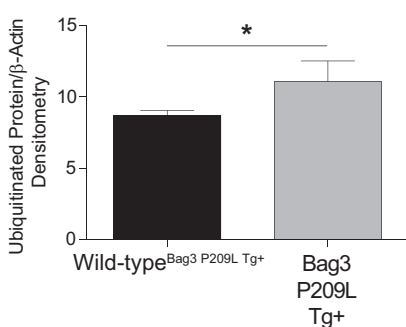

increase in fibrosis, relatively mild heart failure with minimal death (only one recorded), and no classic fibril deposition by TEM. In contrast, the $\alpha$ MHC-CryAB R120G have $100 \%$ mortality by early adulthood, considerable fibril deposition, and impaired myofibril alignment. ${ }^{74}$ These differences may have only to do with the amount of protein present, secondary to its solubility and relationship to interacting proteins they chaperone, because the native small heat shock proteins Bag3 and CryAB have considerably different roles in the adult heart. This issue with the concentration of the mutant protein is significant, as recent studies overexpressing the Bag3 P209L mutant protein in cells result in dramatic increases in aggregated protein in cell culture, ${ }^{75}$ in contrast to the present study in vivo having only onefold to twofold increase in Bag3 P209L and a slower clinical course as seen in the human disease. Recent studies have found that Bag 3 knockdown in adult zebrafish results in myofibrillar disintegration without forming protein aggregates, ${ }^{13}$ whereas $\mathrm{CryAB}^{-1-}$ (and CryAB/HSPB2 double knockout) mice do not have any ultrastructural abnormalities. ${ }^{76}$ The ability of the Bag3 P209L mutant protein to rescue the BAG3 knockdown myofibillar disarray indicates that its structural functions in the sarcomere are still intact. $^{13}$

The importance of maintaining BAG3 levels is highlighted by the Bag3 knockdown studies in zebrafish, making the transcriptional down-regulation of wild-type BAG3 a critical finding in the present study (Figure 8A). How BAG3 is transcriptionally regulated in the cardiomyocyte is unknown, but multiple studies have identified that Jun proteins (c-Jun, JunB, and JunD), which form AP-1 homodimers (Jun/Jun) or heterodimers with Fos family members, ATF/CREB family members, or JDP family members regulate BAG3 expression in noncardiac cells. ${ }^{77}$ In cancer cells, c-Jun can be found to 
interact with the BAG3 promoter, with less c-Jun occupancy driving a suppression of BAG3. ${ }^{7}$

Misfolded tau proteins caused by truncation mutations have been linked to the activation of mitogen-activated protein kinase signaling. ${ }^{78}$ The p38 activation in Alzheimer disease animal models (ie, $A \beta$ and tau toxicities) can be attenuated with vitamin $\mathrm{E}^{79}$ and by medications that are used to successfully reduce loss of memory impairment in Alzheimer disease (Donepezil, an inhibitor of acetylcholinesterase, attenuates amyloid- $\beta$-induced iNOS, cyclooxygenase- 2 , and the phosphorylation of $\mathrm{p} 38$ as well as $\mathrm{NF}-\kappa \mathrm{B}^{80}$ ). Taken together, these studies illustrate the functional role of PAO-prone amyloid- $\beta$ and tau proteins in activating inflammation via $\mathrm{p} 38$ activation, which is a therapeutic target to attenuate disease progression. Similarly, Bag3 $\mathrm{Tg}^{+}$hearts have both an increase in inflammatory infiltrates (Figure 5, C-E) and trending increases in NF- $\kappa \mathrm{B}$ activity (increased phospho-p65/p65 by Western blot) at 12 months of age (Supplemental Figure S5).

These finding have broader implications in treating PAOmediated cardiac diseases, such as the Bag3 P029L mutation, which currently have no therapies available beyond traditional RAAS-targeted heart failure therapies. Similar to the link between misfolded proteins and p38 activation in the brain, ${ }^{81}$ the cardiac literature suggests a parallel role of p38 in mediating heart failure and may be partially responsible for the cardiac phenotype of the current study. Targeted activation of cardiomyocyte p38 potently induces cardiac hypertrophy and heart failure in vivo. ${ }^{69,82,83}$ In the current study, a clear progressive heart failure ensues in the Bag3 P029L Tg + mice by 8 months of age (Figure 1, D-I), along with cardiac hypertrophy (Figure 2B). Also like the amyloid- $\beta$ and tau protein-induced p38 mediatedinflammation, preliminary evidence of inflammation was seen in the 12-month Bag3 P029L Tg + hearts (Figure 5, B and C). ${ }^{70}$ Evidence is accumulating that p38 inhibitors are effective in protecting in cardiac ischemia, arrhythmias, myocardial contractile dysfunction, myocardial infarction, LV remodeling, atherosclerosis, and endothelial cell dysfunction in both preclinical models and in human clinical trials (eg, losmapimod/NCT02145468 in phase 3 for acute MI and BMS-582949/NCT00570752 in phase 1 trials for atherosclerosis) with potential benefit identified in patients with early and late atherosclerosis. ${ }^{84}$ To our knowledge, p38 inhibitors have not been reported in hereditary myopathies to date, but their use may provide a therapy that has shown promise in other cardiac diseases, for which a specific mechanistically rationale therapy is currently unavailable.

\section{Acknowledgments}

We thank Robert Bagnell and Vicky Madden (Microscopy Services Laboratory, UNC Department of Pathology and Laboratory Medicine) for their assistance with the electron microscopy experiments and Carolyn Spaniel (Hillhouse), for her management of the Bag3 P209L colony.
M.S.W. is the guarantor of this work and, as such, had full access to all of the data in the study and takes responsibility for the integrity of the data and the accuracy of the data analysis.

\section{Supplemental Data}

Supplemental material for this article can be found at http://dx.doi.org/10.1016/j.ajpath.2016.03.017.

\section{References}

1. Rosati A, Graziano V, De Laurenzi V, Pascale M, Turco MC: BAG3: a multifaceted protein that regulates major cell pathways. Cell Death Dis 2011, 2:e141

2. Hishiya A, Kitazawa T, Takayama S: BAG3 and Hsc70 interact with actin capping protein CapZ to maintain myofibrillar integrity under mechanical stress. Circ Res 2010, 107:1220-1231

3. Sanoudou D, Corbett MA, Han M, Ghoddusi M, Nguyen MA, Vlahovich N, Hardeman EC, Beggs AH: Skeletal muscle repair in a mouse model of nemaline myopathy. Hum Mol Genet 2006, 15: 2603-2612

4. Warren GL, Summan M, Gao X, Chapman R, Hulderman T, Simeonova PP: Mechanisms of skeletal muscle injury and repair revealed by gene expression studies in mouse models. J Physiol 2007, 582:825-841

5. Olive M, Kley RA, Goldfarb LG: Myofibrillar myopathies: new developments. Curr Opin Neurol 2013, 26:527-535

6. Arimura T, Ishikawa T, Nunoda S, Kawai S, Kimura A: Dilated cardiomyopathy-associated BAG3 mutations impair Z-disc assembly and enhance sensitivity to apoptosis in cardiomyocytes. Hum Mutat 2011, 32:1481-1491

7. Lee HC, Cherk SW, Chan SK, Wong S, Tong TW, Ho WS, Chan AY, Lee KC, Mak CM: BAG3-related myofibrillar myopathy in a Chinese family. Clin Genet 2012, 81:394-398

8. Norton N, Li D, Rieder MJ, Siegfried JD, Rampersaud E, Zuchner S, Mangos S, Gonzalez-Quintana J, Wang L, McGee S, Reiser J, Martin E, Nickerson DA, Hershberger RE: Genome-wide studies of copy number variation and exome sequencing identify rare variants in BAG3 as a cause of dilated cardiomyopathy. Am J Hum Genet 2011, $88: 273-282$

9. Odgerel Z, Sarkozy A, Lee HS, McKenna C, Rankin J, Straub V, Lochmuller H, Paola F, D’Amico A, Bertini E, Bushby K, Goldfarb LG: Inheritance patterns and phenotypic features of myofibrillar myopathy associated with a BAG3 mutation. Neuromuscul Disord 2010, 20:438-442

10. Selcen D, Muntoni F, Burton BK, Pegoraro E, Sewry C, Bite AV, Engel AG: Mutation in BAG3 causes severe dominant childhood muscular dystrophy. Ann Neurol 2009, 65:83-89

11. Homma S, Iwasaki M, Shelton GD, Engvall E, Reed JC, Takayama S: BAG3 deficiency results in fulminant myopathy and early lethality. Am J Pathol 2006, 169:761-773

12. McCollum AK, Casagrande G, Kohn EC: Caught in the middle: the role of Bag3 in disease. Biochem J 2010, 425:e1-e3

13. Ruparelia AA, Oorschot V, Vaz R, Ramm G, Bryson-Richardson RJ: Zebrafish models of BAG3 myofibrillar myopathy suggest a toxic gain of function leading to BAG3 insufficiency. Acta Neuropathol 2014, 128:821-833

14. Willis MS, Patterson C: Proteotoxicity and cardiac dysfunction: Alzheimer's disease of the heart? N Engl J Med 2013, 368:455-464

15. Subramanian K, Gianni D, Balla C, Assenza GE, Joshi M, Semigran MJ, Macgillivray TE, Van Eyk JE, Agnetti G, Paolocci N, Bamburg JR, Agrawal PB, Del Monte F: Cofilin-2 phosphorylation and sequestration in myocardial aggregates: novel pathogenetic 
mechanisms for idiopathic dilated cardiomyopathy. J Am Coll Cardiol 2015, 65:1199-1214

16. Pattison JS, Sanbe A, Maloyan A, Osinska H, Klevitsky R, Robbins J: Cardiomyocyte expression of a polyglutamine preamyloid oligomer causes heart failure. Circulation 2008, 117:2743-2751

17. Sanbe A, Osinska H, Saffitz JE, Glabe CG, Kayed R, Maloyan A, Robbins J: Desmin-related cardiomyopathy in transgenic mice: a cardiac amyloidosis. Proc Natl Acad Sci U S A 2004, 101: $10132-10136$

18. Wang X, Klevitsky R, Huang W, Glasford J, Li F, Robbins J: AlphaB-crystallin modulates protein aggregation of abnormal desmin. Circ Res 2003, 93:998-1005

19. Hayashi M, Imanaka-Yoshida K, Yoshida T, Wood M, Fearns C, Tatake RJ, Lee JD: A crucial role of mitochondrial Hsp40 in preventing dilated cardiomyopathy. Nat Med 2006, 12:128-132

20. Willis MS, Wadosky KM, Rodriguez JE, Schisler JC, Lockyer P, Hilliard EG, Glass DJ, Patterson C: Muscle ring finger 1 and muscle ring finger 2 are necessary but functionally redundant during developmental cardiac growth and regulate E2F1-mediated gene expression in vivo. Cell Biochem Funct 2014, 32:39-50

21. Willis MS, Dyer LA, Ren R, Lockyer P, Moreno-Miralles I, Schisler JC, Patterson C: BMPER regulates cardiomyocyte size and vessel density in vivo. Cardiovasc Pathol 2013, 22:228-240

22. Su H, Wang X: Autophagy and p62 in cardiac protein quality control. Autophagy 2011, 7:1382-1383

23. Su H, Li F, Ranek MJ, Wei N, Wang X: COP9 signalosome regulates autophagosome maturation. Circulation 2011, 124:2117-2128

24. Santiago JJ, Dangerfield AL, Rattan SG, Bathe KL, Cunnington RH, Raizman JE, Bedosky KM, Freed DH, Kardami E, Dixon IM: Cardiac fibroblast to myofibroblast differentiation in vivo and in vitro: expression of focal adhesion components in neonatal and adult rat ventricular myofibroblasts. Dev Dyn 2010, 239:1573-1584

25. Yates CC, Krishna P, Whaley D, Bodnar R, Turner T, Wells A: Lack of CXC chemokine receptor 3 signaling leads to hypertrophic and hypercellular scarring. Am J Pathol 2010, 176:1743-1755

26. Sidorova TN, Mace LC, Wells KS, Yermalitskaya LV, Su PF, Shyr Y, Byrne JG, Petracek MR, Greelish JP, Hoff SJ, Ball SK, Glabe CG, Brown NJ, Barnett JV, Murray KT: Quantitative imaging of preamyloid oligomers, a novel structural abnormality, in human atrial samples. J Histochem Cytochem 2014, 62:479-487

27. Roessner U, Wagner C, Kopka J, Trethewey RN, Willmitzer L: Technical advance: simultaneous analysis of metabolites in potato tuber by gas chromatography-mass spectrometry. Plant J 2000, 23: $131-142$

28. Fiehn O, Wohlgemuth G, Scholz M, Kind T, Lee do Y, Lu Y, Moon S, Nikolau B: Quality control for plant metabolomics: reporting MSI-compliant studies. Plant J 2008, 53:691-704

29. Kind T, Wohlgemuth G, Lee do Y, Lu Y, Palazoglu M, Shahbaz S, Fiehn O: FiehnLib: mass spectral and retention index libraries for metabolomics based on quadrupole and time-of-flight gas chromatography/mass spectrometry. Anal Chem 2009, 81:10038-10048

30. Banerjee R, Bultman SJ, Holley D, Hillhouse C, Bain JR, Newgard CB, Muehlbauer MJ, Willis MS: Non-targeted metabolomics of double-mutant cardiomyocytes reveals a novel role for SWI/SNF complexes in metabolic homeostasis. Metabolomics 2015, $11: 1287-1301$

31. Banerjee R, He J, Spaniel C, Quintana MT, Wang ZJ, Bain JR, Newgard CB, Muehlbauer MJ, Willis MS: Non-targeted metabolomics analysis of cardiac Muscle Ring Finger-1 (MuRF1), MuRF2, and MuRF3 in vivo reveals novel and redundant metabolic changes. Metabolomics 2015, 11:312-322

32. Xia J, Mandal R, Sinelnikov IV, Broadhurst D, Wishart DS: MetaboAnalyst 2.0: a comprehensive server for metabolomic data analysis. Nucleic Acids Res 2012, 40:W127-W133

33. Xia J, Psychogios N, Young N, Wishart DS: MetaboAnalyst: a web server for metabolomic data analysis and interpretation. Nucleic Acids Res 2009, 37:W652-W660
34. Rosati A, Ammirante M, Gentilella A, Basile A, Festa M, Pascale M, Marzullo L, Belisario MA, Tosco A, Franceschelli S, Moltedo O, Pagliuca G, Lerose R, Turco MC: Apoptosis inhibition in cancer cells: a novel molecular pathway that involves BAG3 protein. Int J Biochem Cell Biol 2007, 39:1337-1342

35. Pagliuca MG, Lerose R, Cigliano S, Leone A: Regulation by heavy metals and temperature of the human BAG-3 gene, a modulator of Hsp70 activity. FEBS Lett 2003, 541:11-15

36. Rosati A, Di Salle E, Luberto L, Quinto I, Scala G, Turco MC, Pascale M: Identification of a Btk-BAG3 complex induced by oxidative stress. Leukemia 2009, 23:823-824

37. Ammirante M, Rosati A, Arra C, Basile A, Falco A, Festa M, Pascale M, d'Avenia M, Marzullo L, Belisario MA, De Marco M, Barbieri A, Giudice A, Chiappetta G, Vuttariello E, Monaco M, Bonelli P, Salvatore G, Di Benedetto M, Deshmane SL, Khalili K, Turco MC, Leone A: IKK \{gamma protein is a target of BAG3 regulatory activity in human tumor growth. Proc Natl Acad Sci U S A 2010, 107:7497-7502

38. van Spaendonck-Zwarts KY, van Hessem L, Jongbloed JD, de Walle HE, Capetanaki Y, van der Kooi AJ, van Langen IM, van den Berg MP, van Tintelen JP: Desmin-related myopathy. Clin Genet 2011, 80:354-366

39. Ulbricht A, Gehlert S, Leciejewski B, Schiffer T, Bloch W, Hohfeld J: Induction and adaptation of chaperone-assisted selective autophagy CASA in response to resistance exercise in human skeletal muscle. Autophagy 2015, 11:538-546

40. Arndt V, Dick N, Tawo R, Dreiseidler M, Wenzel D, Hesse M, Furst DO, Saftig P, Saint R, Fleischmann BK, Hoch M, Hohfeld J: Chaperone-assisted selective autophagy is essential for muscle maintenance. Curr Biol 2010, 20:143-148

41. Jiao Q, Sanbe A, Zhang X, Liu JP, Minamisawa S: alphaB-Crystallin R120G variant causes cardiac arrhythmias and alterations in the expression of $\mathrm{Ca}(2+)$-handling proteins and endoplasmic reticulum stress in mice. Clin Exp Pharmacol Physiol 2014, 41:589-599

42. Zheng Q, Su H, Ranek MJ, Wang X: Autophagy and p62 in cardiac proteinopathy. Circ Res 2011, 109:296-308

43. Willis MS, Min JN, Wang S, McDonough $H$, Lockyer $P$, Wadosky KM, Patterson C: Carboxyl terminus of Hsp70-interacting protein (CHIP) is required to modulate cardiac hypertrophy and attenuate autophagy during exercise. Cell Biochem Funct 2013, 31: 724-735

44. Marunouchi T, Tanonaka K: Cell death in the cardiac myocyte. Biol Pharm Bull 2015, 38:1094-1097

45. van Empel VP, Bertrand AT, Hofstra L, Crijns HJ, Doevendans PA, De Windt LJ: Myocyte apoptosis in heart failure. Cardiovasc Res 2005, 67:21-29

46. Doong H, Vrailas A, Kohn EC: What's in the "BAG"?-A functional domain analysis of the BAG-family proteins. Cancer Lett 2002, 188: $25-32$

47. Chiappetta G, Ammirante M, Basile A, Rosati A, Festa M, Monaco M, Vuttariello E, Pasquinelli R, Arra C, Zerilli M, Todaro M, Stassi G, Pezzullo L, Gentilella A, Tosco A, Pascale M, Marzullo L, Belisario MA, Turco MC, Leone A: The antiapoptotic protein BAG3 is expressed in thyroid carcinomas and modulates apoptosis mediated by tumor necrosis factor-related apoptosis-inducing ligand. J Clin Endocrinol Metab 2007, 92:1159-1163

48. Liu P, Xu B, Li J, Lu H: BAG3 gene silencing sensitizes leukemic cells to Bortezomib-induced apoptosis. FEBS Lett 2009, 583: 401-406

49. Jacobs AT, Marnett LJ: HSF1-mediated BAG3 expression attenuates apoptosis in 4-hydroxynonenal-treated colon cancer cells via stabilization of anti-apoptotic Bcl-2 proteins. J Biol Chem 2009, 284: 9176-9183

50. Wang HQ, Liu BQ, Gao YY, Meng X, Guan Y, Zhang HY, Du ZX: Inhibition of the JNK signalling pathway enhances proteasome inhibitor-induced apoptosis of kidney cancer cells by suppression of BAG3 expression. Br J Pharmacol 2009, 158:1405-1412 
51. Festa M, Del Valle L, Khalili K, Franco R, Scognamiglio G, Graziano V, De Laurenzi V, Turco MC, Rosati A: BAG3 protein is overexpressed in human glioblastoma and is a potential target for therapy. Am J Pathol 2011, 178:2504-2512

52. Jung SE, Kim YK, Youn DY, Lim MH, Ko JH, Ahn YS, Lee JH: Down-modulation of Bis sensitizes cell death in C6 glioma cells induced by oxygen-glucose deprivation. Brain Res 2010, 1349: $1-10$

53. Cesaro E, Montano G, Rosati A, Crescitelli R, Izzo P, Turco MC, Costanzo P: WT1 protein is a transcriptional activator of the antiapoptotic bag3 gene. Leukemia 2010, 24:1204-1206

54. Bodnar RJ, Rodgers ME, Chen WC, Wells A: Pericyte regulation of vascular remodeling through the CXC receptor 3. Arterioscler Thromb Vasc Biol 2013, 33:2818-2829

55. Avolio E, Rodriguez-Arabaolaza I, Spencer HL, Riu F, Mangialardi G, Slater SC, Rowlinson J, Alvino VV, Idowu OO, Soyombo S, Oikawa A, Swim MM, Kong CH, Cheng H, Jia H, Ghorbel MT, Hancox JC, Orchard CH, Angelini G, Emanueli C, Caputo M, Madeddu P: Expansion and characterization of neonatal cardiac pericytes provides a novel cellular option for tissue engineering in congenital heart disease. J Am Heart Assoc 2015, 4:e002043

56. Wong SP, Rowley JE, Redpath AN, Tilman JD, Fellous TG, Johnson JR: Pericytes, mesenchymal stem cells and their contributions to tissue repair. Pharmacol Ther 2015, 151:107-120

57. Vicart P, Caron A, Guicheney P, Li Z, Prevost MC, Faure A, Chateau D, Chapon F, Tome F, Dupret JM, Paulin D, Fardeau M: A missense mutation in the alphaB-crystallin chaperone gene causes a desmin-related myopathy. Nat Genet 1998, 20:92-95

58. Parry TL, Melehani JH, Ranek MJ, Willis MS: Functional amyloid signaling via the inflammasome, necrosome, and signalosome: new therapeutic targets in heart failure. Front Cardiovasc Med 2015, 2:25

59. Nakamura T, Lipton SA: S-nitrosylation of critical protein thiols mediates protein misfolding and mitochondrial dysfunction in neurodegenerative diseases. Antioxid Redox Signal 2011, 14:1479-1492

60. Nakamura T, Lipton SA: Redox regulation of mitochondrial fission, protein misfolding, synaptic damage, and neuronal cell death: potential implications for Alzheimer's and Parkinson's diseases. Apoptosis 2010, 15:1354-1363

61. Zungu M, Schisler J, Willis MS: All the little pieces: regulation of mitochondrial fusion and fission by ubiquitin and small ubiquitin-like modifer and their potential relevance in the heart. Circ J 2011, 75: $2513-2521$

62. Du ZX, Zhang HY, Meng X, Gao YY, Zou RL, Liu BQ, Guan Y, Wang HQ: Proteasome inhibitor MG132 induces BAG3 expression through activation of heat shock factor 1. J Cell Physiol 2009, 218: 631-637

63. Franceschelli S, Rosati A, Lerose R, De Nicola S, Turco MC, Pascale M: Bag3 gene expression is regulated by heat shock factor 1 . J Cell Physiol 2008, 215:575-577

64. Song S, Kole S, Precht P, Pazin MJ, Bernier M: Activation of heat shock factor 1 plays a role in pyrrolidine dithiocarbamate-mediated expression of the co-chaperone BAG3. Int J Biochem Cell Biol 2010, 42:1856-1863

65. Wang HQ, Meng X, Liu BQ, Li C, Gao YY, Niu XF, Li N, Guan Y, Du ZX: Involvement of JNK and NF-kappaB pathways in lipopolysaccharide (LPS)-induced BAG3 expression in human monocytic cells. Exp Cell Res 2012, 318:16-24

66. Belozerov VE, Ratkovic S, McNeill H, Hilliker AJ, McDermott JC: In vivo interaction proteomics reveal a novel p38 mitogen-activated protein kinase/Rack1 pathway regulating proteostasis in Drosophila muscle. Mol Cell Biol 2014, 34:474-484

67. Kang EB, Kwon IS, Koo JH, Kim EJ, Kim CH, Lee J, Yang CH, Lee YI, Cho IH, Cho JY: Treadmill exercise represses neuronal cell death and inflammation during Abeta-induced ER stress by regulating unfolded protein response in aged presenilin 2 mutant mice. Apoptosis 2013, 18:1332-1347
68. Huang Y, Li X, Wang Y, Wang H, Huang C, Li J: Endoplasmic reticulum stress-induced hepatic stellate cell apoptosis through calcium-mediated JNK/P38 MAPK and Calpain/Caspase-12 pathways. Mol Cell Biochem 2014, 394:1-12

69. Liao P, Georgakopoulos D, Kovacs A, Zheng M, Lerner D, Pu H, Saffitz J, Chien K, Xiao RP, Kass DA, Wang Y: The in vivo role of p38 MAP kinases in cardiac remodeling and restrictive cardiomyopathy. Proc Natl Acad Sci U S A 2001, 98:12283-12288

70. Degousee N, Martindale J, Stefanski E, Cieslak M, Lindsay TF, Fish JE, Marsden PA, Thuerauf DJ, Glembotski CC, Rubin BB: MAP kinase kinase 6-p38 MAP kinase signaling cascade regulates cyclooxygenase-2 expression in cardiac myocytes in vitro and in vivo. Circ Res 2003, 92:757-764

71. Knezevic T, Myers VD, Gordon J, Tilley DG, Sharp TE 3rd, Wang J, Khalili K, Cheung JY, Feldman AM: BAG3: a new player in the heart failure paradigm. Heart Fail Rev 2015, 20:423-434

72. Fuchs M, Poirier DJ, Seguin SJ, Lambert H, Carra S, Charette SJ, Landry J: Identification of the key structural motifs involved in HspB8/HspB6-Bag3 interaction. Biochem J 2010, 425:245-255

73. Meister M, Minoia M, Kanon B, Carra S, Kampinga H: Characterization of the myopathy associated BAG3 P209L mutation. The biology of molecular chaperones: from molecules, organelles and cells to risfolding diseases. European Molecular Biology Organization Conference, May 17-22, 2013, Santa Margherita di Pula, Italy. Poster 275 .

74. Wang X, Osinska H, Klevitsky R, Gerdes AM, Nieman M, Lorenz J, Hewett T, Robbins J: Expression of R120G-alphaB-crystallin causes aberrant desmin and alphaB-crystallin aggregation and cardiomyopathy in mice. Circ Res 2001, 89:84-91

75. Minoia M: Chaperones, Protein Homeostasis \& Protein Aggregation Diseases. Netherlands, University Medical Center Groningen and University of Groningen, 2014

76. Benjamin IJ, Guo Y, Srinivasan S, Boudina S, Taylor RP, Rajasekaran NS, Gottlieb R, Wawrousek EF, Abel ED, Bolli R: CRYAB and HSPB2 deficiency alters cardiac metabolism and paradoxically confers protection against myocardial ischemia in aging mice. Am J Physiol Heart Circ Physiol 2007, 293: H3201-H3209

77. Li C, Li S, Kong DH, Meng X, Zong ZH, Liu BQ, Guan Y, Du ZX, Wang HQ: BAG3 is upregulated by c-Jun and stabilizes JunD. Biochim Biophys Acta 2013, 1833:3346-3354

78. Kovac A, Zilka N, Kazmerova Z, Cente M, Zilkova M, Novak M: Misfolded truncated protein tau induces innate immune response via MAPK pathway. J Immunol 2011, 187:2732-2739

79. Giraldo E, Lloret A, Fuchsberger T, Vina J: Abeta and tau toxicities in Alzheimer's are linked via oxidative stress-induced p38 activation: protective role of vitamin E. Redox Biol 2014, 2:873-877

80. Kim HG, Moon M, Choi JG, Park G, Kim AJ, Hur J, Lee KT, Oh MS: Donepezil inhibits the amyloid-beta oligomer-induced microglial activation in vitro and in vivo. Neurotoxicology 2014, 40: $23-32$

81. Zilka N, Kazmerova Z, Jadhav S, Neradil P, Madari A, Obetkova D, Bugos O, Novak M: Who fans the flames of Alzheimer's disease brains? misfolded tau on the crossroad of neurodegenerative and inflammatory pathways. J Neuroinflammation 2012, 9:47

82. Wang Y, Huang S, Sah VP, Ross J Jr, Brown JH, Han J, Chien KR: Cardiac muscle cell hypertrophy and apoptosis induced by distinct members of the p38 mitogen-activated protein kinase family. J Biol Chem 1998, 273:2161-2168

83. Braz JC, Bueno OF, Liang Q, Wilkins BJ, Dai YS, Parsons S, Braunwart J, Glascock BJ, Klevitsky R, Kimball TF, Hewett TE, Molkentin JD: Targeted inhibition of p38 MAPK promotes hypertrophic cardiomyopathy through upregulation of calcineurin-NFAT signaling. J Clin Invest 2003, 111:1475-1486

84. Martin ED, Bassi R, Marber MS: p38 MAPK in cardioprotection: are we there yet? Br J Pharmacol 2015, 172:2101-2113 